\title{
Uniconazole and nitrogen fertilization trigger photosynthesis and chlorophyll fluorescence, and delay leaf senescence in maize at a high population density
}

\author{
I. AHMAD ${ }^{*, * *,+}$ \\ , S. AHMAD*, M. KAMRAN*, X.N. YANG ${ }^{*}$, F.J. HOU**,, B.P. YANG ${ }^{*}$, R.X. DING*, \\ T. LIU*, and Q.F. HAN ${ }^{*},+$ \\ Key Laboratory of Crop Physio-Ecology and Tillage Science in North-Western Loess Plateau, Ministry \\ of Agriculture, College of Agronomy, Northwest A\&F University, 712100 Yangling, Shaanxi, China* \\ State Key Laboratory of Grassland Agro-Ecosystems, Key Laboratory of Grassland Livestock Industry Innovation, \\ Ministry of Agriculture and Rural Affairs, Engineering Research Center of Grassland Industry, Ministry of \\ Education, College of Pastoral Agriculture Science and Technology, Lanzhou University, 730020 Lanzhou, China**
}

\begin{abstract}
Reducing the leaf senescence rate could improve the grain yield in maize. In the present study, maize seeds were treated with uniconazole at concentrations of 0 and $25 \mathrm{mg} \mathrm{kg}^{-1}$, and nitrogen was applied at 0,150 , and $225 \mathrm{~kg} \mathrm{ha}^{-1}$ to maize crop planted at a density of 105,000 plants ha ${ }^{-1}$. Applying uniconazole and nitrogen reduced the leaf senescence rate by increasing the leaf area per plant, chlorophyll content, net photosynthetic rate, stomatal conductance, and transpiration rate. Uniconazole and nitrogen increased the electron transport rate, photochemical quenching coefficient, variable fluorescence, maximal quantum yield of PSII photochemistry, and effective quantum yield of PSII photochemistry. Uniconazole and nitrogen reduced the intercellular carbon dioxide concentration, nonphotochemical quenching coefficient, and malondialdehyde content but increased the soluble protein content and antioxidant enzyme activities. Applying uniconazole at a concentration of $25 \mathrm{mg} \mathrm{kg}^{-1}$ and nitrogen at a rate of $150 \mathrm{~kg} \mathrm{ha}^{-1}$ obtained the maximum grain yield in maize.
\end{abstract}

Keywords: leaf senescence; maize; nitrogen; plant population; uniconazole.

\section{Introduction}

Due to rapid increases in the world's population and reductions in arable lands, the global demand for maize will increase by $46 \%$ in 2050 (Hubert et al. 2010). Increasing plant populations is an important approach for improving the grain yield in maize but the high density plant populations lead to competition for resources,

\section{Highlights}

- Uniconazole and nitrogen application improved photosynthetic efficiency of maize

- Uniconazole and nitrogen application delayed leaf senescence in maize

- Treatment $\mathrm{U}_{25} \mathrm{~N}_{150}$ obtained higher grain yield in maize
Received 26 October 2020

Accepted 4 February 2021

Published online 16 March 2021

${ }^{+}$Corresponding author

e-mail: irshad88@aup.edu.pk (I. Ahmad)

cyhoufj@1zu.edu.cn (F.J. Hou)

hanqf88@nwafu.edu.cn (Q.F. Han)

Abbreviations: APX - ascorbate peroxidase; CAT - catalase; $C_{\mathrm{i}}$ - intercellular carbon dioxide concentration; $E$ - transpiration rate; ETR - photosynthetic electron transport rate; $\mathrm{F}_{0}$ - minimal fluorescence yield of the dark-adapted state; $\mathrm{F}_{\mathrm{m}}$ - maximum fluorescence yield of the dark-adapted state; $\mathrm{F}_{\mathrm{v}}$ - variable fluorescence; $\mathrm{F}_{\mathrm{v}} / \mathrm{F}_{\mathrm{m}}-$ maximal quantum yield of PSII photochemistry; $g_{\mathrm{s}}-$ stomatal conductance; $\mathrm{MDA}$ - malondialdehyde; $P_{\mathrm{N}}$ - net photosynthetic rate; $\mathrm{POD}$ - peroxidase; $\mathrm{q}_{\mathrm{N}}$ - nonphotochemical quenching coefficient; $\mathrm{q}_{\mathrm{p}}$ - photochemical quenching coefficient; SOD - superoxide dismutase; $\Phi_{\text {PSII }}$ - effective quantum yield of PSII photochemistry.

Acknowledgements: This study was supported by funding from China Postdoctoral Science Foundation (2019M663837), National High Technology Research and Development Programs of China (863 Program, no. 2013AA102902), the special fund for Agro-Scientific Research in the Public Interest (201303104), the 111 Project of Chinese Education Ministry (B12007).

Conflict of interest: The authors declare that they have no conflict of interest. 
thereby resulting in lodging, accelerated leaf senescence, and reduced photosynthetic activity (Xu et al. 2017, Xue et al. 2017, Kamran et al. 2018). The high density plant populations also decrease the photosynthetic efficiency and starch accumulation (Li et al. 2019). Leaf senescence involves a reduction in the leaf area at the bottom layer and eventually the whole plant (Gregersen et al. 2013, Ahmad et al. 2019a). Extending the photosynthetic duration is closely related to leaf senescence (Wu et al. 2012). Genetic and environmental factors (nutrient stress, water, and temperature) regulate leaf senescence in crops (van Doorn and Woltering 2004, Liu et al. 2005, Gregersen 2011, Thomas and Ougham 2015). The green leaf area and its duration have significant effects on the grain yield, and shaded leaves become senescent earlier compared to unshaded leaves (Maddonni et al. 2001, Gregersen et al. 2013). Accelerated leaf senescence leads to a lower grain yield in maize because of nutritional and hormonal signals (Sadras et al. 2000). Thus, reducing accelerated leaf senescence in dense plant populations is important for increasing the production of maize.

Nitrogen $(\mathrm{N})$ is a major component of proteins and nucleic acids, and its application regulates plant growth and development (Dordas and Sioulas 2008, Li et al. 2012). N has a significant effect on leaf senescence traits and patterns (Kitonyo et al. 2018). Deficient or insufficient $\mathrm{N}$ fertilization inhibits the photosynthetic and radiationuse efficiencies to reduce the grain yield, whereas the optimum $\mathrm{N}$ application concentration can increase the grain yield (Shangguan et al. 2000, Dordas and Sioulas 2008, Luo et al. 2018). A previous study suggested that $\mathrm{N}$ has a positive correlation with the photosynthetic efficiency (Sage and Pearcy 1987). Fang et al. (2018a) reported that $\mathrm{N}$ fertilization enhanced the chlorophyll (Chl) content, net photosynthetic rate $\left(P_{\mathrm{N}}\right)$, stomatal conductance $\left(g_{\mathrm{s}}\right)$, transpiration rate $(E)$, and stomatal limitation value of buckwheat in different plant populations. $\mathrm{N}$ also improved the yield and yield components in cotton (Dong et al. 2012). $\mathrm{N}$ is important for enhancing the photosynthetic efficiency and leaf area (Sage and Pearcy 1987, Su et al. 2019). Wu et al. (2019) reported that low N stress significantly reduced the Chl content, $P_{\mathrm{N}}, g_{\mathrm{s}}$, and $E$, as well as increasing the intercellular carbon dioxide concentration $\left(C_{\mathrm{i}}\right)$ in maize cultivars.

Uniconazole (UCZ) is a triazole-type compound and its application can reduce the lodging rate and protect plants from various stresses (Duan et al. 2008, Wang et al. 2015a, Fang et al. 2018b). The application of triazole improved the $P_{\mathrm{N}}, E$, maximal quantum yield of PSII photochemistry $\left(\mathrm{F}_{\mathrm{v}} / \mathrm{F}_{\mathrm{m}}\right)$, photochemical quenching coefficient $\left(\mathrm{q}_{\mathrm{p}}\right)$, and PSII efficiency in herbaceous peony (Xia et al. 2018), as well as enhancing the Chl content and $P_{\mathrm{N}}$ in Cartharanthus roseus, Photinia, Pyracantha, and dwarf Burford Holly (Frymire and Cole 1992, Jaleel et al. 2009). The application of triazole enhanced the grain yield and quality of rice by delaying leaf senescence due to the increased activities of antioxidant enzymes and reduced malondialdehyde (MDA) content (Pan et al. 2013). UCZ can reduce the degradation of Chl and enhance the activities of antioxidant enzymes (Zhang et al. 2007, Wang et al. 2009). Our previous study suggested that UCZ increased the Chl content, soluble protein $(\mathrm{SP})$ content, $P_{\mathrm{N}}$, and leaf area per maize plant in the bottom layer at the grain-filling stage (Ahmad et al. 2019a). Thus, previous research suggests that UCZ has a major effect on the regulation of leaf senescence.

Our previous study suggested that UCZ increased the lodging resistance and grain yield in wheat and maize (Ahmad et al. 2018a,b; 2019b, 2020). We also found that $\mathrm{UCZ}$ reduced leaf senescence in the bottom layer at the grain-filling stage in maize (Ahmad et al. 2019a). We also showed that the application of UCZ at a concentration of $25 \mathrm{mg} \mathrm{kg}^{-1}$ had a great effect on improving the lodging resistance, seed filling, and antioxidant defense system in medium density plant population. Thus, based on our previous studies, we applied $25 \mathrm{mg} \mathrm{kg}^{-1} \mathrm{UCZ}$ and tested three different $\mathrm{N}$ rates in a high density maize plant population $\left(105,000\right.$ plants $\left.\mathrm{ha}^{-1}\right)$. We assessed the effects of UCZ and different $\mathrm{N}$ rates on the chlorophyll content, photosynthetic performance, chlorophyll fluorescence, antioxidant defense mechanism, and leaf senescence rate in maize. Our findings provide important insights into the delaying of leaf senescence and improving the grain yield in high density maize plant populations.

\section{Materials and methods}

Location, treatments, and field management: A field experiment was conducted during 2019 at the Institute of Water Saving Agriculture in Arid Areas of China $\left(34^{\circ} 20^{\prime} \mathrm{N}, 108^{\circ} 04^{\prime} \mathrm{E} ; 466.7 \mathrm{~m}\right.$ a.s.1.). The average sunshine hours at the experimental site was $2,150 \mathrm{~h}$, the annual average temperature was $12-14^{\circ} \mathrm{C}$, and the total annual precipitation was $580.5 \mathrm{~mm}$. During 2019, the total precipitation in the growth season was $541 \mathrm{~mm}$ and the average temperature was $21.8^{\circ} \mathrm{C}$. The nutrient contents of the $0-40-\mathrm{cm}$ soil layer comprised $1.10 \mathrm{~g}$ (total nitrogen) $\mathrm{kg}^{-1}, 56.14 \mathrm{mg}$ (alkaline nitrogen) $\mathrm{kg}^{-1}, 10.61 \mathrm{mg}$ (available phosphorus) $\mathrm{kg}^{-1}$, and $139.19 \mathrm{mg}$ (available potassium) $\mathrm{kg}^{-1}$.

Maize hybrid Zhengdan 958 seeds were obtained from Henan Golden Doll Seed Co. Ltd, China. UCZ (5\% WP) was obtained from Sichuan Guoguang Agri-Chemical Co. Ltd., China. The experiment employed a factorial design and three replicates. The experiment consists of two factors, where factor A was the UCZ concentration, i.e., $0 \mathrm{mg} \mathrm{kg}^{-1}$ $\left(\mathrm{U}_{0}\right)$ and $25 \mathrm{mg} \mathrm{kg}^{-1}\left(\mathrm{U}_{25}\right)$, and factor $\mathrm{B}$ was the nitrogen rate, i.e., $0 \mathrm{~kg} \mathrm{ha}^{-1}\left(\mathrm{~N}_{0}\right.$, no $\mathrm{N}$ application), $150 \mathrm{~kg} \mathrm{ha}^{-1}$ $\left(\mathrm{N}_{150}\right)$, and $225 \mathrm{~kg} \mathrm{ha}^{-1}\left(\mathrm{~N}_{225}\right)$. Thus, the six combinations of treatments comprised $\mathrm{U}_{0} \mathrm{~N}_{0}, \mathrm{U}_{0} \mathrm{~N}_{150}, \mathrm{U}_{0} \mathrm{~N}_{225}, \mathrm{U}_{25} \mathrm{~N}_{0}$, $\mathrm{U}_{25} \mathrm{~N}_{150}$, and $\mathrm{U}_{25} \mathrm{~N}_{225}$. In our previous studies, we tested different UCZ concentrations and their application times in medium density plant populations. Based on our previous findings, we applied $25 \mathrm{mg}(\mathrm{UCZ}) \mathrm{kg}^{-1}$ to the maize seeds and tested three different $\mathrm{N}$ application rates in a high density plant population. The seeds were treated with UCZ solution at concentrations of $0 \mathrm{mg} \mathrm{kg}^{-1}\left(\mathrm{U}_{0}\right.$, distilled water) and $25 \mathrm{mg} \mathrm{kg}^{-1}\left(\mathrm{U}_{25}\right)$ for $12 \mathrm{~h}$ at $25^{\circ} \mathrm{C}$, and air dried before sowing. Each plot was $7 \mathrm{~m}$ long and $7 \mathrm{~m}$ wide $\left(49 \mathrm{~m}^{2}\right)$. The distance between each plot was $1 \mathrm{~m}$. The distance between rows was maintained at $60 \mathrm{~cm}$ and that 
between plants was $15.8 \mathrm{~cm}$. Plots were manually planted with two seeds per mound and thinning was performed at the three-leaf stage (V3) to obtain a plant population with 105,000 plants ha ${ }^{-1}$. Half of the $\mathrm{N}$ (urea, $46 \% \mathrm{~N}$ ) was applied at the time of seed bed preparation and half at the jointing stage. Phosphorus (calcium superphosphate, 16\% $\mathrm{P}_{2} \mathrm{O}_{5}$ ) and potassium (Pudan Red, $52 \% \mathrm{~K}_{2} \mathrm{O}$ ) were applied at sowing at a rate of $150 \mathrm{~kg} \mathrm{ha}^{-1}$. Sowing was conducted on 12 June and harvesting on 7 October in 2019. No irrigation was applied to the treatments during the growing season.

Leaf area per plant was measured at the V3 stage, eightleaf stage (V8), silking stage (R1, occurs about $3 \mathrm{~d}$ after the tasseling stage and silk is visible outside the husk), $15 \mathrm{~d}$ after the silking stage (R1-15), $30 \mathrm{~d}$ after the silking stage (R1-30), and $45 \mathrm{~d}$ after the silking stage (R1-45) in the middle rows, except for five middle rows. Six plants per replicate were sampled in each stage and the leaf area per plant was calculated as follows: leaf area per plant $\left[\mathrm{cm}^{2}\right]=$ leaf length $\times$ leaf width $\times$ correction factor $(0.75)$

Chl $\boldsymbol{a}$ and $\boldsymbol{b}$ contents were determined for the uppermost leaf at V3 and V8, and the ear-leaf at R1, R1-15, R1-30, and R1-45 using six randomly selected plants for each of three replicates. The leaves initially used for obtaining photosynthesis and Chl fluorescence parameter measurements were sampled to determine the Chl content, MDA content, SP content, and antioxidant enzyme activities at V3, V8, R1, and R1-45, but not at R1-15 and R1-30. The Chl $a$ and $b$ contents were assayed according to Zhang et al. (2012). Briefly, $0.1 \mathrm{~g}$ of the middle leaf portion with the midrib removed was ground with liquid nitrogen and then soaked in the dark for $24 \mathrm{~h}$ in $10 \mathrm{~mL}$ of a solution containing $45 \%$ acetone, $45 \%$ ethanol, and $10 \%$ distilled water. The absorbance of the extract was measured at 663 and $646 \mathrm{~nm}$ with an ultraviolet (UV)-spectrophotometer (UV-2600, Shimadzu Instruments, Suzhou, China).

Photosynthetic parameters: At the V3, V8, R1 (silking stage), R3 (milk stage, $20 \mathrm{~d}$ after the silking stage), and R6 (physiological maturity stage) stages, $P_{\mathrm{N}}, g_{\mathrm{s}}, C_{\mathrm{i}}$, and $E$ were measured with a photosynthetic system (LI-6400 $X T, L i$-Cor, Lincoln, NE, USA). Six plants per replicate were selected and $P_{\mathrm{N}}, g_{\mathrm{s}}, C_{\mathrm{i}}$, and $E$ were measured using the fully expanded uppermost leaf at the V3 and V8 stages, and the ear leaves at R1, R3, and R6 on a clear sunny day between 9:30 and 11:30 h. The measurements of $P_{\mathrm{N}}, g_{\mathrm{s}}$, $C_{\mathrm{i}}$, and $E$ were acquired in a system with a leaf chamber temperature of $25^{\circ} \mathrm{C}$ and the carbon dioxide concentration in the leaf chamber was kept at $380 \mu \mathrm{mol}\left(\mathrm{CO}_{2}\right) \mathrm{mol}^{-1}$ by using a $\mathrm{CO}_{2}$ injector with a high-pressure liquid $\mathrm{CO}_{2}$ cartridge source. The photosynthetic active radiation was set at $1,500 \mu \mathrm{mol}$ (photon) $\mathrm{m}^{-2} \mathrm{~s}^{-1}$ (Wei et al. 2016). The external humidity was $40-50 \%$.

The Chl fluorescence parameters were measured for the uppermost six expanded leaves per replicate at the V3 and V8 stages, and six ear leaves per replicate at the R1, R3, and R6 stages by using a portable chlorophyll fluorometer (FMS 2.02, Hansatech Instruments Ltd.,
United Kingdom). The leaves initially used for measuring the photosynthetic parameters were employed to determine the $\mathrm{Chl}$ fluorescence parameters. The minimal fluorescence $\left(\mathrm{F}_{0}\right)$ was determined for 60 -min darkadapted leaves and the maximum fluorescence $\left(\mathrm{F}_{\mathrm{m}}\right)$ after a saturation light pulse $\left[\left(8,000 \mu \mathrm{mol}(\right.\right.$ photon $\left.\left.) \mathrm{m}^{-2} \mathrm{~s}^{-1}\right)\right]$ of $0.8 \mathrm{~s}$ with the same leaves. The photosynthetic electron transport rate (ETR), $\mathrm{q}_{\mathrm{p}}$, nonphotochemical quenching coefficient $\left(\mathrm{q}_{\mathrm{N}}\right)$, variable fluorescence, maximal quantum yield of PSII photochemistry, and effective quantum yield of PSII photochemistry were determined according to previously described methods (Zivcak et al. 2013, Mu et al. 2010).

MDA and SP contents and antioxidant enzyme activities: The MDA and SP contents and antioxidant enzyme activities were determined with six leaves initially used for analyzing the Chl contents at V3, V8, R1, R1-15, R1-30, and R1-45. First, $0.5 \mathrm{~g}$ of each leaf sample was homogenized in $5 \mathrm{~mL}$ of a pre-cooled mixture comprising $50 \mathrm{mmol} \mathrm{L}^{-1}$ phosphate buffer ( $\left.\mathrm{pH} 7.8\right), 0.1 \mathrm{mM}$ EDTA$\mathrm{Na}_{2}$, and $1 \%(\mathrm{w} / \mathrm{w})$ insoluble polyvinyl pyrrolidone (PVP), and then centrifuged at $15,000 \times g$ for $10 \mathrm{~min}$ at $4^{\circ} \mathrm{C}$. The supernatant was used for determining the MDA content, SP content, and antioxidant enzyme activities.

The MDA content was assayed according to Zhao et al. (2007), where $1 \mathrm{~mL}$ of extract was mixed with $2 \mathrm{~mL}$ of $0.67 \%$ thiobarbituric acid (TBA), heated at $100^{\circ} \mathrm{C}$ for $15 \mathrm{~min}$ in a water bath, and then centrifuged at 4,000 $\times g$ for $15 \mathrm{~min}$ after cooling. The absorbance of the supernatant was measured at 600,532 , and $450 \mathrm{~nm}$ with an UV-spectrophotometer (UV-2600, Shimadzu Instruments, Suzhou, China). The MDA content was expressed as $\mu \mathrm{mol} \mathrm{g}$ (FM).

The SP content was assayed according to Bradford (1976) and the result was expressed as $\mathrm{mg} \mathrm{g}^{-1}$ (FM).

The activity of superoxide dismutase (SOD, EC 1.15.1.1) was determined according to Giannopolitis and Ries (1977), where $20 \mu \mathrm{L}$ of extract was mixed with $3 \mathrm{~mL}$ of SOD reaction mixture $\left(1.5 \mathrm{~mL}\right.$ of $50 \mathrm{mmol} \mathrm{L}^{-1}$ phosphate buffer ( $\mathrm{pH} 7.8$ ), $0.3 \mathrm{~mL}$ of $0.75 \mathrm{mmol} \mathrm{L}^{-1}$ nitroblue tetrazolium, $0.3 \mathrm{~mL}$ of $130 \mathrm{mmol} \mathrm{L}^{-1}$ methionine, $0.3 \mathrm{~mL}$ of $0.02 \mathrm{mmol} \mathrm{L}^{-1}$ riboflavin, $0.3 \mathrm{~mL}$ of $0.1 \mathrm{mmol}$ $\mathrm{L}^{-1}$ EDTA-Na $\mathrm{N}_{2}$, and $0.25 \mathrm{~mL}$ distilled water). The control and enzyme solution were placed in an incubator for $30 \mathrm{~min}$ at $5.86 \mathrm{~W} \mathrm{~m}^{-2}$ and the blank was kept in the dark. The absorbance was determined at $560 \mathrm{~nm}$ with an UV-spectrophotometer (UV-2600, Shimadzu Instruments, Suzhou, China).

The catalase (CAT, EC 1.11.1.6) activity was assayed according to Aebi (1984), where $50 \mu \mathrm{L}$ of extract was mixed with $2.5 \mathrm{~mL}$ of CAT reaction mixture [0.1 mmol $\mathrm{L}^{-1}$ phosphate buffer ( $\mathrm{pH}$ 7.0) and $0.1 \mathrm{mmol} \mathrm{L} \mathrm{H}^{-1} \mathrm{H}_{2} \mathrm{O}_{2}$. The absorbance was measured at $240 \mathrm{~nm}$ with an UVspectrophotometer $(U V-2600$, Shimadzu Instruments, Suzhou, China).

The peroxidase (POD, EC 1.11.1.7) activity was assayed according to Hernández et al. (2000), where $20 \mu \mathrm{L}$ of the extract was mixed with $3 \mathrm{~mL}$ of POD reaction solution [0.1 mmol L $\mathrm{m}^{-1}$ phosphate buffer ( $\mathrm{pH} 6.0$ ), $16 \mathrm{mmol} \mathrm{L}^{-1}$ 
guaiacol, and $19 \mu \mathrm{L}$ of $10 \%(\mathrm{w} / \mathrm{v}) \mathrm{H}_{2} \mathrm{O}_{2}$ ]. The absorbance was measured at $470 \mathrm{~nm}$ with an UV-spectrophotometer (UV-2600, Shimadzu Instruments, Suzhou, China).

The activity of ascorbate peroxidase (APX, EC 1.11.1.11) was determined according to Nakano and Asada (1981), where $0.2 \mathrm{~mL}$ of the extract was mixed with APX reaction mixture $[50 \mathrm{mM}$ phosphate buffer $(\mathrm{pH} 7.0)$, $0.1 \mathrm{mM}$ EDTA, $0.5 \mathrm{mM}$ AsA, and $\left.1.0 \mathrm{mM} \mathrm{H}_{2} \mathrm{O}_{2}\right]$. The change in the absorbance was measured at $290 \mathrm{~nm}$ with an UV-spectrophotometer (UV-2600, Shimadzu Instruments, Suzhou, China).

Ear length, ear diameter, and grain yield: The grain yield was measured at maturity after harvesting the five central rows and avoiding the border rows in each of the three replicates. All of the plants were harvested from the five central rows in each replicate. The cobs were removed from the husk and threshed after $7 \mathrm{~d}$ to measure the grain yield. The ear length and ear diameter were measured based on ten ears per replicate. The ear length was measured with a measuring tape and the ear diameter using a digital Vernier caliper.

Statistical analysis: Analysis of variance ( $A N O V A)$ was performed using Statistix 8.1, where the data obtained from each sampling event and three replicates were analyzed separately. Comparisons of means were performed with the least significant difference (LSD) test at $P<0.05$.

\section{Results}

Leaf area per plant: UCZ and N significantly affected the leaf area per plant under a high density plant population (Table 1). The leaf area per plant tended to increase from the V3 stage and the maximum was reached at R1, then was gradually decreasing until R1-45. Increasing the $\mathrm{N}$ rate increased the leaf area per plant but decreased with
$225 \mathrm{~kg}(\mathrm{~N}) \mathrm{ha}^{-1}$ because the high $\mathrm{N}$ rate increased the plant height and reduced the leaf area per plant. The application of UCZ under different $\mathrm{N}$ rates significantly increased the leaf area per plant, and the maximum leaf area per plant was obtained with $\mathrm{U}_{25} \mathrm{~N}_{150}$ in all growth stages, followed by $\mathrm{U}_{0} \mathrm{~N}_{150}$. The lowest leaf area per plant in all growth stages was obtained with $\mathrm{U}_{0} \mathrm{~N}_{0} . \mathrm{U}_{0} \mathrm{~N}_{150}, \mathrm{U}_{0} \mathrm{~N}_{225}, \mathrm{U}_{25} \mathrm{~N}_{0}$, $\mathrm{U}_{25} \mathrm{~N}_{150}$, and $\mathrm{U}_{25} \mathrm{~N}_{225}$ increased the leaf area per plant at the V3 (by 94, 67, 11, 116, and 78\%, respectively), V8 (by $151,99,16,181$, and 120\%), R1 (by 113, 94, 23, 124, and 98\%), R1-15 (by 145, 110, 31, 162, and 126\%), R1-30 (by $187,147,42,206$, and 166\%), and R1-45 (by 201, 160, 37, 226 , and $180 \%$ ) stages compared to $\mathrm{U}_{0} \mathrm{~N}_{0}$.

Chl $\boldsymbol{a}$ and $\boldsymbol{b}$ contents: The application of UCZ and N significantly increased the $\mathrm{Chl} a$ and $b$ contents at various growth stages (Table 2). The Chl $a$ and $b$ contents increased from $\mathrm{V} 3$ and the maximum occurred at R1, before decreasing until R1-45. Under $\mathrm{U}_{0} \mathrm{~N}_{0}$ and $\mathrm{U}_{25} \mathrm{~N}_{0}$, the maximum $\mathrm{Chl} a$ and $b$ contents were found at $\mathrm{V} 8$, before decreasing until R1-45. Higher $\mathrm{N}$ rates decreased the Chl $a$ and $b$ contents, whereas the optimum $\mathrm{N}$ rate of $150 \mathrm{~kg} \mathrm{ha}^{-1}$ yielded higher Chl $a$ and $b$ contents. The application of $\mathrm{UCZ}$ and $\mathrm{N}$ together increased the Chl $a$ and $b$ contents compared with the application of $\mathrm{N}$ alone. The minimum Chl $a$ and $b$ contents at all growth stages were obtained with $\mathrm{U}_{0} \mathrm{~N}_{0}$ and the maximum with $\mathrm{U}_{25} \mathrm{~N}_{150}$ followed by $\mathrm{U}_{0} \mathrm{~N}_{150}$. The mean results for the six growth stages showed that compared with $\mathrm{U}_{0} \mathrm{~N}_{0}$, treatments $\mathrm{U}_{0} \mathrm{~N}_{150}, \mathrm{U}_{0} \mathrm{~N}_{225}$, $\mathrm{U}_{25} \mathrm{~N}_{0}, \mathrm{U}_{25} \mathrm{~N}_{150}$, and $\mathrm{U}_{25} \mathrm{~N}_{225}$ significantly increased the Chl $a$ contents by $2.44,1.81,0.46,2.73$, and $2.07 \mathrm{mg} \mathrm{g}^{-1}$, respectively, and the $\mathrm{Chl} b$ contents by $0.52,0.34,0.09$, 0.66 , and $0.42 \mathrm{mg} \mathrm{g}^{-1}$.

Photosynthetic parameters: UCZ and N significantly affected $P_{\mathrm{N}}, g_{\mathrm{s}}, C_{\mathrm{i}}$, and $E$ under highly dense plant population (Tables 3,4$)$. UCZ and N significantly increased

Table 1. Effects of uniconazole (UCZ) and nitrogen fertilization on leaf area per plant at three-leaf stage (V3), eight-leaf stage (V8), silking stage (R1), and 15 (R1-15), 30 (R1-30), and 45 (R1-45) d after the silking stage in maize under high density plant population. $* *$ - significant difference at $1 \%$ probability level; $*$ - significant difference at $5 \%$ probability level; ns - no significant difference. Values are the means based on three replicates. Different lowercase letters within a column indicate significant differences at $P \leq 0.05$ (LSD test). $\mathrm{U}_{0} \mathrm{~N}_{0}\left[0 \mathrm{mg}(\mathrm{UCZ}) \mathrm{kg}^{-1}+0 \mathrm{~kg}(\mathrm{~N}) \mathrm{ha}^{-1}\right] ; \mathrm{U}_{0} \mathrm{~N}_{150}\left[0 \mathrm{mg}(\mathrm{UCZ}) \mathrm{kg}^{-1}+150 \mathrm{~kg}(\mathrm{~N}) \mathrm{ha}^{-1}\right] ; \mathrm{U}_{0} \mathrm{~N}_{225}\left[0 \mathrm{mg}(\mathrm{UCZ}) \mathrm{kg}^{-1}+225 \mathrm{~kg}(\mathrm{~N}) \mathrm{ha}^{-1}\right] ;$ $\mathrm{U}_{25} \mathrm{~N}_{0}\left[25 \mathrm{mg}(\mathrm{UCZ}) \mathrm{kg}^{-1}+0 \mathrm{~kg}(\mathrm{~N}) \mathrm{ha}^{-1}\right] ; \mathrm{U}_{25} \mathrm{~N}_{150}\left[25 \mathrm{mg}(\mathrm{UCZ}) \mathrm{kg}^{-1}+150 \mathrm{~kg}(\mathrm{~N}) \mathrm{ha}^{-1}\right] ; \mathrm{U}_{25} \mathrm{~N}_{225}\left[25 \mathrm{mg}(\mathrm{UCZ}) \mathrm{kg}^{-1}+225 \mathrm{~kg}(\mathrm{~N}) \mathrm{ha}{ }^{-1}\right]$.

\begin{tabular}{llcccccc}
\hline UCZ & \multirow{2}{*}{ Nitrogen } & \multicolumn{2}{c}{ Leaf area per plant $\left[\mathrm{cm}^{2}\right]$} \\
& \multicolumn{2}{c}{$\mathrm{V} 3$} & $\mathrm{~V} 8$ & $\mathrm{R} 1$ & $\mathrm{R} 1-15$ & $\mathrm{R} 1-30$ & $\mathrm{R} 1-45$ \\
\hline $\mathrm{U}_{0}$ & $\mathrm{~N}_{0}$ & $64^{\mathrm{d}}$ & $941^{\mathrm{f}}$ & $3,053^{\mathrm{e}}$ & $2,532^{\mathrm{f}}$ & $2,005^{\mathrm{f}}$ & $1,707^{\mathrm{f}}$ \\
$\mathrm{U}_{0}$ & $\mathrm{~N}_{150}$ & $123^{\mathrm{b}}$ & $2,362^{\mathrm{b}}$ & $6,512^{\mathrm{b}}$ & $6,200^{\mathrm{b}}$ & $5,761^{\mathrm{b}}$ & $5,143^{\mathrm{b}}$ \\
$\mathrm{U}_{0}$ & $\mathrm{~N}_{225}$ & $106^{\mathrm{c}}$ & $1,874^{\mathrm{d}}$ & $5,924^{\mathrm{c}}$ & $5,323^{\mathrm{d}}$ & $4,943^{\mathrm{d}}$ & $4,430^{\mathrm{d}}$ \\
$\mathrm{U}_{25}$ & $\mathrm{~N}_{0}$ & $71^{\mathrm{d}}$ & $1,087^{\mathrm{e}}$ & $3,767^{\mathrm{d}}$ & $3,323^{\mathrm{e}}$ & $2,856^{\mathrm{e}}$ & $2,343^{\mathrm{e}}$ \\
$\mathrm{U}_{25}$ & $\mathrm{~N}_{150}$ & $137^{\mathrm{a}}$ & $2,647^{\mathrm{a}}$ & $6,834^{\mathrm{a}}$ & $6,634^{\mathrm{a}}$ & $6,134^{\mathrm{a}}$ & $5,563^{\mathrm{a}}$ \\
$\mathrm{U}_{25}$ & $\mathrm{~N}_{225}$ & $113^{\mathrm{c}}$ & $2,070^{\mathrm{c}}$ & $6,045^{\mathrm{c}}$ & $5,734^{\mathrm{c}}$ & $5,342^{\mathrm{c}}$ & $4,785^{\mathrm{c}}$ \\
ANOVA & & & & & & & \\
$\mathrm{UCZ}$ & & $* *$ & $* *$ & $* *$ & $* *$ & $* *$ & $* *$ \\
$\mathrm{~N}$ & & $* *$ & $* *$ & $* *$ & $* *$ & $* *$ & $* *$ \\
$\mathrm{UCZ} \times \mathrm{N}$ & & $\mathrm{ns}$ & $\mathrm{ns}$ & $* *$ & $* *$ & $* *$ & $* *$ \\
\hline
\end{tabular}


Table 2. Effects of uniconazole (UCZ) and nitrogen fertilization on chlorophyll (Chl) $a$ and Chl $b$ content at the three-leaf stage (V3), eight-leaf stage (V8), silking stage (R1), and 15 (R1-15), 30 (R1-30), and 45 (R1-45) d after the silking stage in maize under high density plant population. ${ }^{* *}$ - significant difference at $1 \%$ probability level; $*$ - significant difference at $5 \%$ probability level; ns - no significant difference. Values are the means based on three replicates. Different lowercase letters within a column indicate significant differences at $P \leq 0.05$ (LSD test). Abbreviations of the treatment names are the same as those given in Table 1.

\begin{tabular}{|c|c|c|c|c|c|c|c|c|c|c|c|c|c|}
\hline \multirow[t]{2}{*}{$\mathrm{UCZ}$} & \multirow[t]{2}{*}{ Nitrogen } & \multicolumn{6}{|c|}{ Chl $a\left[\mathrm{mg} \mathrm{g}^{-1}(\mathrm{FM})\right]$} & \multicolumn{6}{|c|}{ Chl $b\left[\mathrm{mg} \mathrm{g}^{-1}(\mathrm{FM})\right]$} \\
\hline & & V3 & V8 & $\mathrm{R} 1$ & $\mathrm{R} 1-15$ & $\mathrm{R} 1-30$ & R1-45 & V3 & V8 & $\mathrm{R} 1$ & $\mathrm{R} 1-15$ & $\mathrm{R} 1-30$ & $\mathrm{R} 1-45$ \\
\hline $\mathrm{U}_{0}$ & $\mathrm{~N}_{0}$ & $2.36^{\mathrm{e}}$ & $4.34^{\mathrm{e}}$ & $3.41^{\mathrm{e}}$ & $2.71^{\mathrm{f}}$ & $2.10^{\mathrm{f}}$ & $0.61^{\mathrm{e}}$ & $0.46^{\mathrm{e}}$ & $0.86^{\mathrm{d}}$ & $0.70^{\mathrm{e}}$ & $0.58^{\mathrm{d}}$ & $0.10^{\mathrm{d}}$ & $0.08^{\mathrm{f}}$ \\
\hline $\mathrm{U}_{0}$ & $\mathrm{~N}_{150}$ & $4.02^{\mathrm{ab}}$ & $5.44^{\mathrm{b}}$ & $6.41^{\mathrm{a}}$ & $5.82^{\mathrm{b}}$ & $5.38^{\mathrm{b}}$ & $3.12^{\mathrm{b}}$ & $0.76^{\mathrm{b}}$ & $1.13^{\mathrm{b}}$ & $1.34^{\mathrm{b}}$ & $1.21^{\mathrm{b}}$ & $0.90^{\mathrm{a}}$ & $0.56^{\mathrm{b}}$ \\
\hline $\mathrm{U}_{0}$ & $\mathrm{~N}_{225}$ & $3.29^{c}$ & $4.89^{c}$ & $5.81^{\mathrm{c}}$ & $5.15^{\mathrm{d}}$ & $4.61^{\mathrm{d}}$ & $2.62^{\mathrm{c}}$ & $0.63^{\mathrm{c}}$ & $1.00^{\mathrm{c}}$ & $1.13^{\mathrm{c}}$ & $1.06^{\mathrm{c}}$ & $0.72^{\mathrm{b}}$ & $0.28^{\mathrm{d}}$ \\
\hline $\mathrm{U}_{25}$ & $\mathrm{~N}_{0}$ & $2.61^{\mathrm{d}}$ & $4.57^{\mathrm{d}}$ & $3.85^{\mathrm{d}}$ & $3.45^{\mathrm{e}}$ & $3.01^{\mathrm{e}}$ & $0.81^{\mathrm{d}}$ & $0.51^{\mathrm{d}}$ & $0.91^{\mathrm{d}}$ & $0.84^{\mathrm{d}}$ & $0.65^{\mathrm{d}}$ & $0.25^{\mathrm{c}}$ & $0.14^{\mathrm{e}}$ \\
\hline $\mathrm{U}_{25}$ & $\mathrm{~N}_{150}$ & $4.19^{\mathrm{a}}$ & $5.91^{\mathrm{a}}$ & $6.53^{\mathrm{a}}$ & $6.01^{\mathrm{a}}$ & $5.74^{\mathrm{a}}$ & $3.53^{\mathrm{a}}$ & $0.82^{\mathrm{a}}$ & $1.21^{\mathrm{a}}$ & $1.59^{\mathrm{a}}$ & $1.39^{\mathrm{a}}$ & $0.96^{\mathrm{a}}$ & $0.76^{\mathrm{a}}$ \\
\hline $\mathrm{U}_{25}$ & $\mathrm{~N}_{225}$ & $3.88^{\mathrm{b}}$ & $5.01^{\mathrm{c}}$ & $6.04^{\mathrm{d}}$ & $5.34^{\mathrm{c}}$ & $5.01^{\mathrm{c}}$ & $2.68^{\mathrm{c}}$ & $0.74^{\mathrm{b}}$ & $1.04^{\mathrm{c}}$ & $1.23^{\mathrm{bc}}$ & $1.06^{\mathrm{c}}$ & $0.76^{\mathrm{b}}$ & $0.47^{\mathrm{c}}$ \\
\hline \multicolumn{14}{|l|}{$A N O V A$} \\
\hline UCZ & & $* *$ & $* *$ & $* *$ & $* *$ & $* *$ & $* *$ & $* *$ & $* *$ & $* *$ & $*$ & $* *$ & $* *$ \\
\hline $\mathrm{N}$ & & $* *$ & $* *$ & $* *$ & $* *$ & $* *$ & $* *$ & $* *$ & $* *$ & $* *$ & $* *$ & $* *$ & $* *$ \\
\hline $\mathrm{UCZ} \times \mathrm{N}$ & & $* *$ & $*$ & $*$ & $* *$ & $* *$ & $* *$ & ns & ns & ns & ns & ns & $* *$ \\
\hline
\end{tabular}

Table 3. Effects of uniconazole (UCZ) and nitrogen fertilization on net photosynthetic rate $\left(P_{\mathrm{N}}\right)$ and stomatal conductance $\left(g_{\mathrm{s}}\right)$ at the three-leaf stage (V3), eight-leaf stage (V8), silking stage (R1), milk stage (R3), and physiological maturity stage (R6) in maize under high density plant population. $* *$ - significant difference at $1 \%$ probability level; $*$ - significant difference at $5 \%$ probability level; ns no significant difference. Values are the means based on three replicates. Different lowercase letters within a column indicate significant differences at $P \leq 0.05$ (LSD test). Abbreviations of the treatment names are the same as those given in Table 1.

\begin{tabular}{|c|c|c|c|c|c|c|c|c|c|c|c|}
\hline \multirow[t]{2}{*}{$\mathrm{UCZ}$} & \multirow[t]{2}{*}{ Nitrogen } & \multicolumn{5}{|c|}{$P_{\mathrm{N}}\left[\mu \mathrm{mol} \mathrm{m}{ }^{-2} \mathrm{~s}^{-1}\right]$} & \multicolumn{5}{|c|}{$g_{\mathrm{s}}\left[\mathrm{mol}\left(\mathrm{H}_{2} \mathrm{O}\right) \mathrm{m}^{-2} \mathrm{~s}^{-1}\right]$} \\
\hline & & V3 & V8 & $\mathrm{R} 1$ & $\mathrm{R} 3$ & R6 & V3 & V8 & $\mathrm{R} 1$ & $\mathrm{R} 3$ & R6 \\
\hline $\mathrm{U}_{0}$ & $\mathrm{~N}_{0}$ & $17.2^{\mathrm{f}}$ & $21.4^{\mathrm{e}}$ & $19.4^{\mathrm{f}}$ & $15.8^{\mathrm{e}}$ & $6.0^{\mathrm{e}}$ & $0.08^{\mathrm{e}}$ & $0.14^{\mathrm{e}}$ & $0.18^{\mathrm{d}}$ & $0.09^{\mathrm{d}}$ & $0.08^{\mathrm{c}}$ \\
\hline $\mathrm{U}_{0}$ & $\mathrm{~N}_{150}$ & $27.8^{\mathrm{b}}$ & $32.3^{\mathrm{b}}$ & $40.6^{\mathrm{b}}$ & $35.3^{\mathrm{a}}$ & $18.1^{\mathrm{b}}$ & $0.18^{\mathrm{b}}$ & $0.24^{\mathrm{b}}$ & $0.29^{\mathrm{ab}}$ & $0.22^{\mathrm{ab}}$ & $0.16^{\mathrm{ab}}$ \\
\hline $\mathrm{U}_{0}$ & $\mathrm{~N}_{225}$ & $22.2^{\mathrm{d}}$ & $27.1^{\mathrm{c}}$ & $34.7^{\mathrm{d}}$ & $29.7^{\mathrm{c}}$ & $16.3^{\mathrm{c}}$ & $0.16^{\mathrm{c}}$ & $0.19^{\mathrm{cd}}$ & $0.24^{\mathrm{c}}$ & $0.18^{\mathrm{c}}$ & $0.13^{\mathrm{b}}$ \\
\hline $\mathrm{U}_{25}$ & $\mathrm{~N}_{0}$ & $19.0^{\mathrm{e}}$ & $24.7^{\mathrm{d}}$ & $22.1^{\mathrm{e}}$ & $18.4^{\mathrm{d}}$ & $8.8^{\mathrm{d}}$ & $0.12^{\mathrm{d}}$ & $0.16^{\mathrm{de}}$ & $0.20^{\mathrm{d}}$ & $0.11^{\mathrm{d}}$ & $0.09^{\mathrm{c}}$ \\
\hline $\mathrm{U}_{25}$ & $\mathrm{~N}_{150}$ & $29.3^{\mathrm{a}}$ & $34.8^{\mathrm{a}}$ & $43.2^{\mathrm{a}}$ & $37.1^{\mathrm{a}}$ & $22.1^{\mathrm{a}}$ & $0.22^{\mathrm{a}}$ & $0.28^{\mathrm{a}}$ & $0.32^{\mathrm{a}}$ & $0.24^{\mathrm{a}}$ & $0.18^{\mathrm{a}}$ \\
\hline $\mathrm{U}_{25}$ & $\mathrm{~N}_{225}$ & $24.2^{\mathrm{c}}$ & $28.9^{c}$ & $37.5^{\mathrm{c}}$ & $31.9^{\mathrm{b}}$ & $17.4^{\mathrm{bc}}$ & $0.18^{\mathrm{b}}$ & $0.21^{\mathrm{bc}}$ & $0.26^{\mathrm{bc}}$ & $0.20^{\mathrm{bc}}$ & $0.14^{\mathrm{b}}$ \\
\hline \multicolumn{12}{|l|}{$A N O V A$} \\
\hline $\mathrm{UCZ}$ & & $* *$ & $* *$ & $* *$ & $* *$ & $* *$ & $* *$ & $* *$ & $*$ & $* *$ & ns \\
\hline $\mathrm{N}$ & & $* *$ & $* *$ & $* *$ & $* *$ & $* *$ & $* *$ & $* *$ & $* *$ & $* *$ & $* *$ \\
\hline $\mathrm{UCZ} \times \mathrm{N}$ & & ns & ns & ns & ns & ns & ns & ns & ns & ns & ns \\
\hline
\end{tabular}

$P_{\mathrm{N}}, g_{\mathrm{s}}$, and $E$ at all growth stages, but decreased $C_{\mathrm{i}} . P_{\mathrm{N}}, g_{\mathrm{s}}$. $E$ increased from the V3 stage and the maximum values were reached at R1, then it was decreasing until R6. $C_{\mathrm{i}}$ increased from the $\mathrm{V} 3$ stage and the maximum values were reached at R6. Under $\mathrm{U}_{0} \mathrm{~N}_{0}$ and $\mathrm{U}_{25} \mathrm{~N}_{0}$, the maximum $P_{\mathrm{N}}$ values were reached at $\mathrm{V} 8$, then were gradually decreasing until R6. Increasing the $\mathrm{N}$ rate increased $P_{\mathrm{N}}, g_{\mathrm{s}}$, and $E$ but the higher $\mathrm{N}$ rate of $225 \mathrm{~kg} \mathrm{ha}^{-1}$ decreased $P_{\mathrm{N}}, g_{\mathrm{s}}$, and $E$. The application of UCZ together with $\mathrm{N}$ obtained higher $P_{\mathrm{N}}, g_{\mathrm{s}}$, and $E$ values but decreased $C_{\mathrm{i}}$ compared with the application of $\mathrm{N}$ alone. The maximum $P_{\mathrm{N}}, g_{\mathrm{s}}$, and $E$ values and minimum $C_{\mathrm{i}}$ values were obtained under $\mathrm{U}_{25} \mathrm{~N}_{150}$ followed by $\mathrm{U}_{0} \mathrm{~N}_{150}$, whereas the minimum $P_{\mathrm{N}}, g_{\mathrm{s}}$, and $E$ values and maximum $C_{\mathrm{i}}$ values were recorded under $\mathrm{U}_{0} \mathrm{~N}_{0}$. Our results suggest that the application of $\mathrm{UCZ}$ together with $\mathrm{N}$ significantly increased the $\mathrm{Chl}$ content to enhance the photosynthetic capacity of maize plants. The mean results in five growth stages showed that compared with $\mathrm{U}_{0} \mathrm{~N}_{0}$, treatments $\mathrm{U}_{0} \mathrm{~N}_{150}, \mathrm{U}_{0} \mathrm{~N}_{225}, \mathrm{U}_{25} \mathrm{~N}_{0}, \mathrm{U}_{25} \mathrm{~N}_{150}$, and $\mathrm{U}_{25} \mathrm{~N}_{225}$ increased $P_{\mathrm{N}}$ by $93.1,62.9,16.5,108.6$, and $75.3 \%$, respectively, $g_{\mathrm{s}}$ by $91.2,57.9,19.3,117.5$, and $73.7 \%$, and $E$ by $74.8,42.5,12.6,95.3$, and $56.7 \%$, but decreased $C_{\mathrm{i}}$ by $107.3,63.3,21.8,138.1$, and $84.9 \%$.

Chl fluorescence: The application of UCZ and N significantly affected ETR, $\mathrm{q}_{\mathrm{p}}, \mathrm{q}_{\mathrm{N}}, \mathrm{F}_{\mathrm{v}} / \mathrm{F}_{\mathrm{m}}, \Phi_{\mathrm{PSII}}$, and $\mathrm{F}_{\mathrm{v}}$ in the high density plant population (Tables $5,6,7$ ). UCZ and N increasedETR, $\mathrm{q}_{\mathrm{p}}, \mathrm{F}_{\mathrm{v}} / \mathrm{F}_{\mathrm{m}}, \Phi_{\mathrm{PSII}}$, and $\mathrm{F}_{\mathrm{v}}$ but decreased $\mathrm{q}_{\mathrm{N}}$. ETR, $\mathrm{q}_{\mathrm{p}}$, and $\Phi_{\mathrm{PSII}}$ increased from the $\mathrm{V} 3$ stage and the maximum values were reached at $\mathrm{R} 1$, then were decreasing until R6. Under $\mathrm{U}_{0} \mathrm{~N}_{0}$ and $\mathrm{U}_{25} \mathrm{~N}_{0}, E T R, \mathrm{q}_{\mathrm{p}}$, and $\Phi_{\text {PSII }}$ increased from the V3 stage and the peak values were reached at the V8 stage, then decreased. $\mathrm{q}_{\mathrm{N}}$ was the highest at the V3 stage but it then decreased at the V8 stage, before increasing 
Table 4. Effects of uniconazole (UCZ) and nitrogen fertilization on intercellular $\mathrm{CO}_{2}$ concentration $\left(C_{\mathrm{i}}\right)$ and transpiration rate $(E)$ at the three-leaf stage (V3), eight-leaf stage (V8), silking stage (R1), milk stage (R3), and physiological maturity stage (R6) in maize under high density plant population. $* *$ - significant difference at $1 \%$ probability level; $*$ - significant difference at $5 \%$ probability level; ns no significant difference. Values are the means based on three replicates. Different lowercase letters within a column indicate significant differences at $P \leq 0.05$ (LSD test). Abbreviations of the treatment names are the same as those given in Table 1.

\begin{tabular}{|c|c|c|c|c|c|c|c|c|c|c|c|}
\hline \multirow[t]{2}{*}{ UCZ } & \multirow[t]{2}{*}{ Nitrogen } & \multicolumn{5}{|c|}{$C_{\mathrm{i}}\left[\mathrm{mol}\left(\mathrm{CO}_{2}\right) \mathrm{m}^{-2} \mathrm{~s}^{-1}\right]$} & \multicolumn{5}{|c|}{$E\left[\mathrm{mmol}\left(\mathrm{H}_{2} \mathrm{O}\right) \mathrm{m}^{-2} \mathrm{~s}^{-1}\right]$} \\
\hline & & V3 & V8 & $\mathrm{R} 1$ & R3 & R6 & V3 & V8 & $\mathrm{R} 1$ & R3 & R6 \\
\hline $\mathrm{U}_{0}$ & $\mathrm{~N}_{0}$ & $74^{\mathrm{a}}$ & $159^{\mathrm{a}}$ & $187^{\mathrm{a}}$ & $203^{\mathrm{a}}$ & $370^{\mathrm{a}}$ & $2.6^{\mathrm{e}}$ & $3.1^{\mathrm{e}}$ & $3.5^{\mathrm{e}}$ & $2.2^{\mathrm{e}}$ & $1.3^{\mathrm{d}}$ \\
\hline $\mathrm{U}_{0}$ & $\mathrm{~N}_{150}$ & $37^{\mathrm{de}}$ & $64^{\mathrm{e}}$ & $81^{\mathrm{d}}$ & $115^{\mathrm{e}}$ & $182^{\mathrm{d}}$ & $4.6^{\mathrm{b}}$ & $5.7^{\mathrm{ab}}$ & $6.4^{\mathrm{ab}}$ & $3.5^{\mathrm{ab}}$ & $2.0^{\mathrm{ab}}$ \\
\hline $\mathrm{U}_{0}$ & $\mathrm{~N}_{225}$ & $47^{c}$ & $87^{\mathrm{c}}$ & $112^{\mathrm{c}}$ & $145^{\mathrm{c}}$ & $217^{c}$ & $3.1^{\mathrm{d}}$ & $5.0^{c}$ & $5.6^{\mathrm{c}}$ & $2.9^{\text {cd }}$ & $1.5^{\mathrm{cd}}$ \\
\hline $\mathrm{U}_{25}$ & $\mathrm{~N}_{0}$ & $67^{\mathrm{b}}$ & $129^{b}$ & $157^{\mathrm{b}}$ & $178^{\mathrm{b}}$ & $284^{\mathrm{b}}$ & $2.1^{\mathrm{f}}$ & $3.8^{\mathrm{d}}$ & $4.4^{\mathrm{d}}$ & $2.5^{\mathrm{de}}$ & $1.5^{\mathrm{cd}}$ \\
\hline $\mathrm{U}_{25}$ & $\mathrm{~N}_{150}$ & $33^{\mathrm{e}}$ & $53^{\mathrm{f}}$ & $69^{\mathrm{e}}$ & $98^{f}$ & $164^{\mathrm{e}}$ & $5.5^{\mathrm{a}}$ & $6.3^{\mathrm{a}}$ & $7.0^{\mathrm{a}}$ & $3.8^{\mathrm{a}}$ & $2.2^{\mathrm{a}}$ \\
\hline $\mathrm{U}_{25}$ & $\mathrm{~N}_{225}$ & $40^{\mathrm{d}}$ & $75^{\mathrm{d}}$ & $102^{\mathrm{c}}$ & $130^{\mathrm{d}}$ & $190^{\mathrm{d}}$ & $3.7^{\mathrm{c}}$ & $5.3^{\mathrm{bc}}$ & $6.1^{\mathrm{bc}}$ & $3.1^{\mathrm{bc}}$ & $1.7^{\mathrm{bc}}$ \\
\hline \multicolumn{12}{|l|}{$A N O V A$} \\
\hline UCZ & & $* *$ & $* *$ & $* *$ & $* *$ & $* *$ & $*$ & $* *$ & $* *$ & ns & $*$ \\
\hline $\mathrm{N}$ & & $* *$ & $* *$ & $* *$ & $* *$ & $* *$ & $* *$ & $* *$ & $* *$ & $* *$ & $* *$ \\
\hline $\mathrm{UCZ} \times \mathrm{N}$ & & ns & $* *$ & $*$ & ns & $* *$ & $* *$ & ns & $\mathrm{ns}$ & ns & ns \\
\hline
\end{tabular}

Table 5. Effects of uniconazole (UCZ) and nitrogen fertilization on photosynthetic electron transport rate (ETR) and photochemical quenching coefficient $\left(\mathrm{q}_{\mathrm{p}}\right)$ at the three-leaf stage (V3), eight-leaf stage (V8), silking stage (R1), milk stage (R3), and physiological maturity stage (R6) in maize under high density plant population. ** - significant difference at $1 \%$ probability level; * - significant difference at $5 \%$ probability level; ns - no significant difference. Values are the means based on three replicates. Different lowercase letters within a column indicate significant differences at P0.05 (LSD test). Abbreviations of the treatment names are the same as those given in Table 1.

\begin{tabular}{|c|c|c|c|c|c|c|c|c|c|c|c|}
\hline \multirow[t]{2}{*}{ UCZ } & \multirow[t]{2}{*}{ Nitrogen } & \multicolumn{5}{|l|}{ ETR } & \multicolumn{5}{|l|}{$\mathrm{q}_{\mathrm{p}}$} \\
\hline & & V3 & V8 & $\mathrm{R} 1$ & R3 & R6 & V3 & V8 & $\mathrm{R} 1$ & R3 & R6 \\
\hline $\mathrm{U}_{0}$ & $\mathrm{~N}_{0}$ & $106^{\mathrm{d}}$ & $121^{\mathrm{e}}$ & $98^{f}$ & $85^{\mathrm{f}}$ & $56^{\mathrm{f}}$ & $0.23^{\mathrm{d}}$ & $0.29^{\mathrm{d}}$ & $0.27^{\mathrm{d}}$ & $0.23^{c}$ & $0.15^{\mathrm{f}}$ \\
\hline $\mathrm{U}_{0}$ & $\mathrm{~N}_{150}$ & $147^{\mathrm{ab}}$ & $164^{\mathrm{b}}$ & $172^{\mathrm{b}}$ & $149^{\mathrm{b}}$ & $90^{\mathrm{b}}$ & $0.30^{\mathrm{ab}}$ & $0.39^{\mathrm{ab}}$ & $0.45^{\mathrm{a}}$ & $0.41^{\mathrm{a}}$ & $0.28^{\mathrm{b}}$ \\
\hline $\mathrm{U}_{0}$ & $\mathrm{~N}_{225}$ & $131^{\mathrm{c}}$ & $147^{c}$ & $152^{\mathrm{d}}$ & $127^{\mathrm{d}}$ & $79^{d}$ & $0.27^{\mathrm{c}}$ & $0.35^{\mathrm{c}}$ & $0.39^{\mathrm{b}}$ & $0.35^{\mathrm{b}}$ & $0.23^{\mathrm{d}}$ \\
\hline $\mathrm{U}_{25}$ & $\mathrm{~N}_{0}$ & $113^{\mathrm{d}}$ & $132^{\mathrm{d}}$ & $115^{\mathrm{e}}$ & $95^{\mathrm{e}}$ & $62^{\mathrm{e}}$ & $0.24^{\mathrm{d}}$ & $0.32^{\mathrm{d}}$ & $0.30^{\mathrm{c}}$ & $0.25^{\mathrm{c}}$ & $0.17^{\mathrm{e}}$ \\
\hline $\mathrm{U}_{25}$ & $\mathrm{~N}_{150}$ & $156^{\mathrm{a}}$ & $169^{a}$ & $179^{\mathrm{a}}$ & $162^{\mathrm{a}}$ & $95^{\mathrm{a}}$ & $0.32^{\mathrm{a}}$ & $0.41^{\mathrm{a}}$ & $0.47^{\mathrm{a}}$ & $0.43^{\mathrm{a}}$ & $0.33^{\mathrm{a}}$ \\
\hline $\mathrm{U}_{25}$ & $\mathrm{~N}_{225}$ & $138^{\mathrm{bc}}$ & $149^{c}$ & $161^{\mathrm{c}}$ & $137^{\mathrm{c}}$ & $83^{c}$ & $0.29^{\mathrm{bc}}$ & $0.37^{\mathrm{bc}}$ & $0.41^{\mathrm{b}}$ & $0.37^{b}$ & $0.24^{\mathrm{c}}$ \\
\hline \multicolumn{12}{|l|}{ ANOVA } \\
\hline UCZ & & $*$ & $*$ & $* *$ & $* *$ & $* *$ & $*$ & $*$ & $* *$ & $*$ & $* *$ \\
\hline $\mathrm{N}$ & & $* *$ & $* *$ & $* *$ & $* *$ & $* *$ & $* *$ & $* *$ & $* *$ & $* *$ & $* *$ \\
\hline $\mathrm{UCZ} \times \mathrm{N}$ & & ns & ns & $*$ & ns & ns & ns & ns & $\mathrm{ns}$ & ns & $* *$ \\
\hline
\end{tabular}

from R1 to R6. $F_{v} / F_{m}$ and $\Phi_{\text {PSII }}$ were the highest at the V3 stage but they decreased at the V8 stage, increased again at the R1 stage, and then decreased gradually until maturity. Increasing the $\mathrm{N}$ rate significantly increased ETR, $\mathrm{q}_{\mathrm{p}}, \mathrm{F}_{\mathrm{v}} / \mathrm{F}_{\mathrm{m}}, \Phi_{\mathrm{PSII}}$, and $\mathrm{F}_{\mathrm{v}}$, where $150 \mathrm{~kg}(\mathrm{~N})$ ha $^{-1}$ obtained better results compared to the high $\mathrm{N}$ rate. The application of UCZ together with $\mathrm{N}$ increased ETR, $\mathrm{q}_{\mathrm{p}}, \mathrm{F}_{\mathrm{v}} / \mathrm{F}_{\mathrm{m}}, \Phi_{\mathrm{PSII}}$, and $\mathrm{F}_{\mathrm{v}}$, and decreased $\mathrm{q}_{\mathrm{N}}$ compared to the application of $\mathrm{N}$ alone. The maximum ETR, $\mathrm{q}_{\mathrm{p}}, \mathrm{F}_{\mathrm{v}} / \mathrm{F}_{\mathrm{m}}, \Phi_{\text {PSII }}$, and $\mathrm{F}_{\mathrm{v}}$ values and the lowest $\mathrm{q}_{\mathrm{N}}$ values in all growth stages were obtained with $\mathrm{U}_{25} \mathrm{~N}_{150}$ followed by $\mathrm{U}_{0} \mathrm{~N}_{150}$. $\mathrm{U}_{0} \mathrm{~N}_{0}$ obtained lower ETR, $\mathrm{q}_{\mathrm{p}}, \mathrm{F}_{\mathrm{v}} / \mathrm{F}_{\mathrm{m}}, \Phi_{\mathrm{PSII}}$, and $\mathrm{F}_{\mathrm{v}}$ values and higher $\mathrm{q}_{\mathrm{N}}$ values. The mean results based on five growth stages showed that compared to $\mathrm{U}_{0} \mathrm{~N}_{0}, \mathrm{U}_{0} \mathrm{~N}_{150}, \mathrm{U}_{0} \mathrm{~N}_{225}, \mathrm{U}_{25} \mathrm{~N}_{0}, \mathrm{U}_{25} \mathrm{~N}_{150}$, and $\mathrm{U}_{25} \mathrm{~N}_{225}$ increased ETR by $54.9,36.5,10.9,63.3$, and $43.3 \%$, respectively, $\mathrm{q}_{\mathrm{p}}$ by $56.4,35.9,9.4,67.5$, and $43.6 \%$,
$\mathrm{F}_{\mathrm{v}} / \mathrm{F}_{\mathrm{m}}$ by $16.3,10.3,4.2,18.7$, and $13 \%$, $\Phi_{\mathrm{PSII}}$ by 67.5 , $40.2,12.8,83.8$, and $51.3 \%$, and $F_{v}$ by $31.0,18.6,7.1$, 37.0 , and $23.7 \%$, but reduced $\mathrm{q}_{\mathrm{N}}$ by $11.2,4.9,2.4,16.0$, and $8.4 \%$.

MDA and SP contents: The application of UCZ and N fertilizer reduced the MDA contents and increased the SP contents in the high density plant population (Table $1 \mathrm{~S}$, supplement). The MDA contents increased from V3 to V8, decreased at R1, and then tended to increase until R1-45. The SP contents increased from V3 to V8, decreased at R1, increased until R1-30, and again decreased at R1-45. Compared to the application of $\mathrm{N}$ alone, the application of UCZ together with $\mathrm{N}$ increased the SP contents and decreased the MDA contents. The maximum MDA content in all growth stages were recorded under $\mathrm{U}_{0} \mathrm{~N}_{0}$ 
Table 6. Effects of uniconazole (UCZ) and nitrogen fertilization on nonphotochemical quenching coefficient $\left(\mathrm{q}_{\mathrm{N}}\right)$ and maximal quantum efficiency of PSII photochemistry $\left(\mathrm{F}_{\mathrm{v}} / \mathrm{F}_{\mathrm{m}}\right)$ at the three-leaf stage (V3), eight-leaf stage (V8), silking stage (R1), milk stage (R3), and physiological maturity stage (R6) in maize under high density plant population. $* *$ - significant difference at $1 \%$ probability level; * - significant difference at 5\% probability level; ns - no significant difference. Values are the means based on three replicates. Different lowercase letters within a column indicate significant differences at $P \leq 0.05$ (LSD test). Abbreviations of the treatment names are the same as those given in Table 1.

\begin{tabular}{|c|c|c|c|c|c|c|c|c|c|c|c|}
\hline $\mathrm{UCZ}$ & Nitrogen & $\begin{array}{l}\mathrm{q}_{\mathrm{N}} \\
\mathrm{V} 3\end{array}$ & V8 & R1 & R3 & R6 & $\begin{array}{l}\mathrm{F}_{\mathrm{v}} / \mathrm{F}_{\mathrm{m}} \\
\mathrm{V} 3\end{array}$ & V8 & R1 & R3 & R6 \\
\hline $\mathrm{U}_{0}$ & $\mathrm{~N}_{0}$ & $0.86^{\mathrm{a}}$ & $0.83^{\mathrm{a}}$ & $0.85^{\mathrm{a}}$ & $0.86^{\mathrm{a}}$ & $0.88^{\mathrm{a}}$ & $0.75^{\mathrm{c}}$ & $0.71^{\mathrm{d}}$ & $0.67^{\mathrm{e}}$ & $0.62^{\mathrm{e}}$ & $0.56^{\mathrm{e}}$ \\
\hline $\mathrm{U}_{0}$ & $\mathrm{~N}_{150}$ & $0.81^{\mathrm{c}}$ & $0.76^{\mathrm{bc}}$ & $0.73^{\mathrm{de}}$ & $0.76^{\mathrm{d}}$ & $0.79^{d}$ & $0.80^{\mathrm{ab}}$ & $0.77^{\mathrm{ab}}$ & $0.80^{\mathrm{ab}}$ & $0.76^{\mathrm{ab}}$ & $0.72^{\mathrm{a}}$ \\
\hline $\mathrm{U}_{0}$ & $\mathrm{~N}_{225}$ & $0.83^{\mathrm{b}}$ & $0.81^{\mathrm{a}}$ & $0.80^{\mathrm{bc}}$ & $0.81^{\mathrm{bc}}$ & $0.83^{\mathrm{c}}$ & $0.77^{\mathrm{bc}}$ & $0.75^{\mathrm{bc}}$ & $0.76^{\mathrm{c}}$ & $0.71^{\mathrm{c}}$ & $0.66^{c}$ \\
\hline $\mathrm{U}_{25}$ & $\mathrm{~N}_{0}$ & $0.84^{\mathrm{b}}$ & $0.81^{\mathrm{a}}$ & $0.83^{\mathrm{ab}}$ & $0.84^{\mathrm{ab}}$ & $0.86^{\mathrm{b}}$ & $0.77^{\mathrm{bc}}$ & $0.73^{\mathrm{cd}}$ & $0.70^{\mathrm{d}}$ & $0.66^{d}$ & $0.59^{d}$ \\
\hline $\mathrm{U}_{25}$ & $\mathrm{~N}_{150}$ & $0.78^{\mathrm{d}}$ & $0.73^{\mathrm{c}}$ & $0.70^{\mathrm{e}}$ & $0.72^{\mathrm{e}}$ & $0.76^{\mathrm{e}}$ & $0.81^{\mathrm{a}}$ & $0.78^{\mathrm{a}}$ & $0.82^{\mathrm{a}}$ & $0.79^{\mathrm{a}}$ & $0.73^{\mathrm{a}}$ \\
\hline $\mathrm{U}_{25}$ & $\mathrm{~N}_{225}$ & $0.80^{\mathrm{c}}$ & $0.79^{\mathrm{ab}}$ & $0.77^{\mathrm{cd}}$ & $0.79^{\text {cd }}$ & $0.80^{\mathrm{d}}$ & $0.78^{\mathrm{abc}}$ & $0.76^{\mathrm{ab}}$ & $0.78^{\mathrm{bc}}$ & $0.74^{\mathrm{bc}}$ & $0.68^{b}$ \\
\hline \multicolumn{12}{|l|}{$A N O V A$} \\
\hline $\mathrm{UCZ}$ & & $* *$ & $\mathrm{~ns}$ & $* *$ & * & $* *$ & ns & ns & $* *$ & $* *$ & $* *$ \\
\hline $\mathrm{N}$ & & $* *$ & $* *$ & $* *$ & $* *$ & $* *$ & $* *$ & $* *$ & $* *$ & $* *$ & $* *$ \\
\hline $\mathrm{UCZ} \times \mathrm{N}$ & & ns & ns & $\mathrm{ns}$ & ns & ns & ns & ns & ns & ns & ns \\
\hline
\end{tabular}

Table 7. Effects of uniconazole (UCZ) and nitrogen fertilization on effective quantum yield of PSII photochemistry $\left(\Phi_{\text {PSII }}\right)$ and variable fluorescence $\left(F_{v}\right)$ at the three-leaf stage (V3), eight-leaf stage (V8), silking stage (R1), milk stage (R3), and physiological maturity stage (R6) in maize under high density plant population. $* *$ - significant difference at $1 \%$ probability level; * - significant difference at $5 \%$ probability level; ns - no significant difference. Values are the means based on three replicates. Different lowercase letters within a column indicate significant differences at $P \leq 0.05$ (LSD test). Abbreviations of the treatment names are the same as those given in Table 1.

\begin{tabular}{|c|c|c|c|c|c|c|c|c|c|c|c|}
\hline \multirow[t]{2}{*}{ UCZ } & \multirow[t]{2}{*}{ Nitrogen } & \multicolumn{5}{|l|}{$\Phi_{\text {PSII }}$} & \multicolumn{5}{|l|}{$\mathrm{F}_{\mathrm{v}}$} \\
\hline & & V3 & V8 & $\mathrm{R} 1$ & $\mathrm{R} 3$ & R6 & V3 & V8 & $\mathrm{R} 1$ & $\mathrm{R} 3$ & R6 \\
\hline $\mathrm{U}_{0}$ & $\mathrm{~N}_{0}$ & $0.22^{\mathrm{c}}$ & $0.29^{\mathrm{d}}$ & $0.27^{\mathrm{f}}$ & $0.23^{\mathrm{e}}$ & $0.16^{\mathrm{f}}$ & $0.92^{\mathrm{c}}$ & $0.89^{\mathrm{d}}$ & $0.82^{\mathrm{d}}$ & $0.73^{\mathrm{e}}$ & $0.61^{\mathrm{f}}$ \\
\hline $\mathrm{U}_{0}$ & $\mathrm{~N}_{150}$ & $0.29^{\mathrm{ab}}$ & $0.40^{\mathrm{ab}}$ & $0.53^{\mathrm{b}}$ & $0.42^{\mathrm{b}}$ & $0.32^{\mathrm{b}}$ & $1.15^{\mathrm{a}}$ & $1.07^{\mathrm{ab}}$ & $1.11^{\mathrm{ab}}$ & $1.01^{\mathrm{ab}}$ & $0.86^{\mathrm{b}}$ \\
\hline $\mathrm{U}_{0}$ & $\mathrm{~N}_{225}$ & $0.27^{\mathrm{b}}$ & $0.37^{\mathrm{b}}$ & $0.42^{\mathrm{d}}$ & $0.33^{\mathrm{cd}}$ & $0.25^{\mathrm{d}}$ & $1.05^{\mathrm{b}}$ & $0.99^{c}$ & $1.02^{\mathrm{c}}$ & $0.89^{c}$ & $0.76^{\mathrm{d}}$ \\
\hline $\mathrm{U}_{25}$ & $\mathrm{~N}_{0}$ & $0.23^{\mathrm{c}}$ & $0.33^{\mathrm{c}}$ & $0.30^{\mathrm{e}}$ & $0.27^{\mathrm{de}}$ & $0.19^{\mathrm{e}}$ & $1.01^{\mathrm{b}}$ & $0.92^{\mathrm{d}}$ & $0.87^{\mathrm{d}}$ & $0.80^{\mathrm{d}}$ & $0.65^{\mathrm{e}}$ \\
\hline $\mathrm{U}_{25}$ & $\mathrm{~N}_{150}$ & $0.31^{\mathrm{a}}$ & $0.42^{\mathrm{a}}$ & $0.56^{\mathrm{a}}$ & $0.51^{\mathrm{a}}$ & $0.35^{\mathrm{a}}$ & $1.19^{\mathrm{a}}$ & $1.10^{\mathrm{a}}$ & $1.16^{\mathrm{a}}$ & $1.07^{\mathrm{a}}$ & $0.92^{\mathrm{a}}$ \\
\hline $\mathrm{U}_{25}$ & $\mathrm{~N}_{225}$ & $0.28^{\mathrm{b}}$ & $0.38^{\mathrm{b}}$ & $0.46^{\mathrm{c}}$ & $0.38^{\mathrm{bc}}$ & $0.27^{\mathrm{c}}$ & $1.05^{\mathrm{b}}$ & $1.03^{\mathrm{bc}}$ & $1.06^{\mathrm{bc}}$ & $0.96^{\mathrm{b}}$ & $0.81^{\mathrm{c}}$ \\
\hline \multicolumn{12}{|l|}{ ANOVA } \\
\hline UCZ & & $\mathrm{ns}$ & $*$ & $* *$ & $* *$ & $* *$ & $*$ & $*$ & $* *$ & $* *$ & $* *$ \\
\hline $\mathrm{N}$ & & $* *$ & $* *$ & $* *$ & $* *$ & $* *$ & $* *$ & $* *$ & $* *$ & $* *$ & $* *$ \\
\hline $\mathrm{UCZ} \times \mathrm{N}$ & & $\mathrm{ns}$ & $\mathrm{ns}$ & ns & $\mathrm{ns}$ & ns & ns & $\mathrm{ns}$ & ns & ns & ns \\
\hline
\end{tabular}

and the minimum under $\mathrm{U}_{25} \mathrm{~N}_{150}$. The highest SP contents in all growth stages were recorded under $\mathrm{U}_{25} \mathrm{~N}_{150}$ and the minimum under $\mathrm{U}_{0} \mathrm{~N}_{0}$. The mean results based on six growth stages showed that compared to $\mathrm{U}_{0} \mathrm{~N}_{0}, \mathrm{U}_{0} \mathrm{~N}_{150}$, $\mathrm{U}_{0} \mathrm{~N}_{225}, \mathrm{U}_{25} \mathrm{~N}_{0}, \mathrm{U}_{25} \mathrm{~N}_{150}$, and $\mathrm{U}_{25} \mathrm{~N}_{225}$ decreased the MDA contents by $39.7,27.6,12.6,62.9$, and $42 \%$, respectively, and increased the SP contents by 33.1, 22, 6.7, 57.4, and $33.5 \%$.

Antioxidant enzyme activities: The antioxidant enzyme activities increased significantly by $\mathrm{UCZ}$ and $\mathrm{N}$ in the high density plant population (Tables $2 \mathrm{~S}, 3 \mathrm{~S}$, supplement). The activity of SOD increased from V3 to R1-15 and then decreased. The activity of POD increased from V3 to R1-30 and then decreased. The activities of CAT and APX were the highest at V3, decreased at V8, and then increased from R1 until R1-45. Increasing N enhanced the antioxidant enzyme activities but they decreased when $\mathrm{N}$ was applied at $225 \mathrm{~kg} \mathrm{ha}^{-1}$. The application of UCZ together with $\mathrm{N}$ obtained higher antioxidant enzyme activities compared to the application of $\mathrm{N}$ alone. The minimum antioxidant enzyme activities were recorded under $\mathrm{U}_{0} \mathrm{~N}_{0}$ and the maximum activities under $\mathrm{U}_{25} \mathrm{~N}_{150}$. The mean results based on six growth stages showed that compared to $\mathrm{U}_{0} \mathrm{~N}_{0}, \mathrm{U}_{0} \mathrm{~N}_{150}, \mathrm{U}_{0} \mathrm{~N}_{225}, \mathrm{U}_{25} \mathrm{~N}_{0}, \mathrm{U}_{25} \mathrm{~N}_{150}$, and $\mathrm{U}_{25} \mathrm{~N}_{225}$ increased the activities of SOD by $37,27.2,8.8$, 49.7, and $35.8 \%$, respectively, POD by 44.7, 33.2, 10.4, 63.6, and $47.0 \%$, CAT by $46.3,31.8,9.7,71.2$, and $55.4 \%$, and APX by 32.8, 23.3, 8.6, 52.7, and 38.2\%. The higher antioxidant enzyme activities significantly protected the photosynthetic system and increased the grain yield in maize. 
Table 8. Effects of uniconazole (UCZ) and nitrogen fertilization on ear length, ear diameter, and grain yield in maize under high density plant population. ${ }^{* *}$ - significant difference at $1 \%$ probability level; ${ }^{*}$ - significant difference at $5 \%$ probability level; ns - no significant difference. Values are the means based on three replicates. Different lowercase letters within a column indicate significant differences at $P \leq 0.05$ (LSD test). Abbreviations of the treatment names are the same as those given in Table 1.

\begin{tabular}{lllll}
\hline $\mathrm{UCZ}$ & Nitrogen & Ear length $[\mathrm{cm}]$ & Ear diameter $[\mathrm{mm}]$ & Grain yield $\left[\mathrm{kg} \mathrm{ha}^{-1}\right]$ \\
\hline $\mathrm{U}_{0}$ & $\mathrm{~N}_{0}$ & $10.6^{\mathrm{e}}$ & $38.9^{\mathrm{d}}$ & $4,134^{\mathrm{e}}$ \\
$\mathrm{U}_{0}$ & $\mathrm{~N}_{150}$ & $16.0^{\mathrm{b}}$ & $48.2^{\mathrm{b}}$ & $10,676^{\mathrm{bc}}$ \\
$\mathrm{U}_{0}$ & $\mathrm{~N}_{225}$ & $14.7^{\mathrm{c}}$ & $46.9^{\mathrm{b}}$ & $10,310^{\mathrm{c}}$ \\
$\mathrm{U}_{25}$ & $\mathrm{~N}_{0}$ & $11.4^{\mathrm{d}}$ & $41.8^{\mathrm{c}}$ & $5,150^{\mathrm{d}}$ \\
$\mathrm{U}_{25}$ & $\mathrm{~N}_{150}$ & $16.9^{\mathrm{a}}$ & $50.7^{\mathrm{a}}$ & $13,728^{\mathrm{a}}$ \\
$\mathrm{U}_{25}$ & $\mathrm{~N}_{225}$ & $15.1^{\mathrm{c}}$ & $48.2^{\mathrm{b}}$ & $11,100^{\mathrm{b}}$ \\
ANOVA & & & & \\
$\mathrm{UCZ}$ & & $* *$ & $* *$ & $* *$ \\
$\mathrm{~N}$ & & $* *$ & $* *$ & $* *$ \\
$\mathrm{UCZ} \times \mathrm{N}$ & & $\mathrm{ns}$ & $\mathrm{ns}$ & $* *$ \\
\hline
\end{tabular}

Ear length, ear diameter, and grain yield: The application of UCZ and N significantly increased the ear length, ear diameter, and grain yield in maize at a high density plant population (Table 8). Compared to the application of $\mathrm{N}$ alone, applying UCZ together with $\mathrm{N}$ increased the ear length, ear diameter, and grain yield with a high density plant population. The minimum ear length, ear diameter, and grain yield were obtained under $\mathrm{U}_{0} \mathrm{~N}_{0}$ and the maximum values under $\mathrm{U}_{25} \mathrm{~N}_{150}$. Compared to $\mathrm{U}_{0} \mathrm{~N}_{0}$, $\mathrm{U}_{0} \mathrm{~N}_{150}, \mathrm{U}_{0} \mathrm{~N}_{225}, \mathrm{U}_{25} \mathrm{~N}_{0}, \mathrm{U}_{25} \mathrm{~N}_{150}$, and $\mathrm{U}_{25} \mathrm{~N}_{225}$ increased the ear length by $50.9,38.7,7.5,59.4$, and $42.5 \%$, respectively, the ear diameter by $23.9,20.6,7.5,30.3$, and $23.9 \%$, and the grain yield by $158.2,149.4,24.6,232.1$, and $168.5 \%$.

\section{Discussion}

Chl contents, SP contents, photosynthesis, and relationships with leaf senescence: Reducing maize accelerated leaf senescence and protecting the photosynthetic apparatus is important for a high density plant population. Leaf senescence is closely correlated with reductions in the Chl content and leaf area per plant (Wang et al. 2009). Chl degradation reduces the photosynthetic efficiency (Wang et al. 2015b). In our previous study, we found that $\mathrm{UCZ}$ reduced leaf senescence in the bottom layer at the grain-filling stage in maize and increased the Chl contents and $P_{\mathrm{N}}$ in a medium density plant population (Ahmad et al. 2019a). In the present study, we applied $25 \mathrm{mg}(\mathrm{UCZ}) \mathrm{kg}^{-1}$ and different $\mathrm{N}$ rates in the high density plant population. Our results suggested that the leaf area per plant and Chl $a$ and $b$ contents gradually increased from V3 to R1, then were decreasing as the plants became older (Tables 1,2). Under $\mathrm{U}_{0} \mathrm{~N}_{0}$ and $\mathrm{U}_{25} \mathrm{~N}_{0}$, the leaf area per plant, $C h l a$ and $b$ contents, and $P_{\mathrm{N}}$ increased from V3 to V8, then they were decreasing until maturity. Furthermore, the leaf area per plant, Chl $a$ and $b$ contents, SP contents, $P_{\mathrm{N}}, g_{\mathrm{s}}$, and $E$ were significantly lower, whereas $C_{\mathrm{i}}$ was higher under $\mathrm{U}_{0} \mathrm{~N}_{0}$ and $\mathrm{U}_{25} \mathrm{~N}_{0}$. Under $\mathrm{U}_{0} \mathrm{~N}_{0}$ and $\mathrm{U}_{25} \mathrm{~N}_{0}$, leaf senescence started after V8, and thus the Chl contents and photosynthetic efficiency were lower. Applying UCZ together with N increased the leaf area per plant, $\mathrm{Chl} a$ and $b$ contents, SP contents, $P_{\mathrm{N}}, g_{\mathrm{s}}$, and $E$ but decreased $C_{\mathrm{i}}$. UCZ application together with $\mathrm{N}$ increased the leaf area per plant, Chl $a$ and $b$ contents, SP contents, $P_{\mathrm{N}}, g_{\mathrm{s}}$, and $E$ but decreased $C_{\mathrm{i}}$ compared to the application of $\mathrm{N}$ alone. $\mathrm{U}_{25} \mathrm{~N}_{150}$ obtained the maximum leaf area per plant, $\mathrm{Chl} a$ and $b$ contents, SP contents, $P_{\mathrm{N}}, g_{\mathrm{s}}$, and $E$ but decreased $C_{\mathrm{i}}$. Our results suggest that UCZ and N had crucial effects on enhancing the photosynthetic efficiency and crop growth. In the present study, the application of UCZ together with $\mathrm{N}$ at different rates significantly increased the leaf area per plant, Chl contents, and photosynthetic efficiency. UCZ is a plant growth regulator that blocks gibberellic acid biosynthesis by inhibiting the conversion of kaurene to kaurenoic acid (Davis et al. 1988). Furthermore, UCZ inhibits the activity of ent-kaurene oxidase, thereby resulting in the accumulation of geranyl diphosphate and enhancing Chl biosynthesis (Soumya et al. 2017). UCZ alters the endogenous hormone contents by inhibiting gibberellic acid to increase the abscisic acid and cytokinin contents. Our recent study of wheat suggested that UCZ affected the endogenous hormone contents of the flag leaves to increase the $\mathrm{Chl}$ contents and canopy apparent photosynthesis (Ahmad et al. 2021).

Effects of UCZ and $\mathrm{N}$ fertilization on Chl fluorescence parameters: Chl fluorescence is an important component of photosynthesis and it can be measured to evaluate the state of PSII and the yield performance (Wang et al. 2013, Ali et al. 2018). Chl fluorescence increases in newly expanding leaves but decreases significantly as leaf senescence proceeds (Zhong et al. 2019). In our previous study, we did not measure the Chl fluorescence parameters. Thus, in the present study, we measured the Chl fluorescence parameters at five different growth stages in order to determine the effects of UCZ and N on these parameters during the leaf senescence process. Our results suggested that the application of UCZ together with $\mathrm{N}$ significantly affected the Chl fluorescence parameters (Tables 5, 6, 7). In particular, the application of UCZ and 
$\mathrm{N}$ significantly increased ETR, $\mathrm{q}_{\mathrm{p}}, \mathrm{F}_{\mathrm{v}} / \mathrm{F}_{\mathrm{m}}, \Phi_{\text {PSII }}$, and $\mathrm{F}_{\mathrm{v}}$, but decreased $\mathrm{q}_{\mathrm{N}}$ in all growth stages. The application of UCZ with $\mathrm{N}$ resulted in higher ETR, $\mathrm{q}_{\mathrm{p}}, \mathrm{F}_{\mathrm{v}} / \mathrm{F}_{\mathrm{m}}, \Phi_{\mathrm{PSII}}$, and $\mathrm{F}_{\mathrm{v}}$ values but lower $\mathrm{q}_{\mathrm{N}}$ values compared to the application of $\mathrm{N}$ alone. The maximum ETR, $\mathrm{q}_{\mathrm{p}}, \mathrm{F}_{\mathrm{v}} / \mathrm{F}_{\mathrm{m}}, \Phi_{\mathrm{PSII}}$, and $\mathrm{F}_{\mathrm{v}}$ values, and the lowest $\mathrm{q}_{\mathrm{N}}$ values were recorded under $\mathrm{U}_{25} \mathrm{~N}_{150}$, whereas the lowest ETR, $\mathrm{q}_{\mathrm{p}}, \mathrm{F}_{\mathrm{v}} / \mathrm{F}_{\mathrm{m}}, \Phi_{\mathrm{PSII}}$, and $\mathrm{F}_{\mathrm{v}}$ values, and the highest $\mathrm{q}_{\mathrm{N}}$ values were obtained under $\mathrm{U}_{0} \mathrm{~N}_{0}$. It is known that $\mathrm{N}$ deficiency can decrease $\mathrm{q}_{\mathrm{p}}$ and $\mathrm{F}_{\mathrm{v}} / \mathrm{F}_{\mathrm{m}}$ but increase $\mathrm{q}_{\mathrm{N}}$ to affect the photochemical activity of PSII and photosynthesis (Yu et al. 2004). N stress significantly reduces ETR, $F_{\mathrm{v}} / \mathrm{F}_{\mathrm{m}}, \mathrm{q}_{\mathrm{p}}$, and $\Phi_{\mathrm{PSII}}$ but increases $\mathrm{q}_{\mathrm{N}}$ in maize (Wu et al. 2019). Our results indicate that $\mathrm{U}_{0} \mathrm{~N}_{0}$ caused photoinhibition and damaged the potential active center of PSII. Our findings suggest that the application of UCZ together with $\mathrm{N}$ protected the photosynthetic system. $\mathrm{N}$ at a rate of $225 \mathrm{~kg} \mathrm{ha}^{-1}$ decreased ETR, $\mathrm{q}_{\mathrm{p}}, \mathrm{F}_{\mathrm{v}} / \mathrm{F}_{\mathrm{m}}, \Phi_{\text {PSII }}$, and $F_{v}$ but increased $q_{N}$. A previous study showed that a higher $\mathrm{N}$ application rate could increase $\mathrm{q}_{\mathrm{N}}$ but decrease ETR, $\mathrm{q}_{\mathrm{p}}$, and $\mathrm{F}_{\mathrm{v}} / \mathrm{F}_{\mathrm{m}}$ (Shrestha et al. 2012). In addition, the optimum $N$ application rate increased $F_{v}, F_{m}, F_{v} / F_{m}, \Phi_{P S I}$, ETR, and $\mathrm{q}_{\mathrm{p}}$ in naked oats but $\mathrm{F}_{\mathrm{v}} / \mathrm{F}_{\mathrm{m}}, \mathrm{ETR}, \mathrm{q}_{\mathrm{p}}$, and $\Phi_{\text {PSII }}$ decreased in cotton when $\mathrm{N}$ was applied at a high rate (Lin et al. 2013). Previous studies demonstrated that the energy transformation efficiency in the PSII reaction center and photosynthesis increased by the application of plant growth regulators (Wang et al. 2013, Ajigboye et al. 2014). Thus, our findings indicate that the application of UCZ together with $\mathrm{N}$ significantly increased ETR, $\mathrm{q}_{\mathrm{p}}, \mathrm{F}_{\mathrm{v}} / \mathrm{F}_{\mathrm{m}}, \Phi_{\mathrm{PSII}}$, and $\mathrm{F}_{\mathrm{v}}$ but decreased $\mathrm{q}_{\mathrm{N}}$ during the leaf senescence process and protected the photosynthetic system.

Role of MDA accumulation in leaf senescence process: Leaf senescence enhances the MDA contents, which can have detrimental effects on plant growth (Zhang et al. 2010). The increased accumulation of MDA has been detected in the leaf senescence process in various crops (Zhang et al. 2010, Wang et al. 2015b). Our results showed that the MDA contents were lower at R1 and they increased gradually as leaf senescence proceeded. Applying UCZ together with $\mathrm{N}$ decreased the MDA contents during the leaf senescence process. The MDA contents were lower when UCZ was applied together with $\mathrm{N}$ compared to the application of $\mathrm{N}$ alone. Lower MDA contents were obtained in all growth stages under $\mathrm{U}_{25} \mathrm{~N}_{150}$ and the maximum contents were found under $\mathrm{U}_{0} \mathrm{~N}_{0}$. A previous study also found that UCZ reduced the MDA contents (Wang et al. 2009). In addition, our previous study showed that $\mathrm{UCZ}$ reduced the MDA contents in wheat and maize (Ahmad et al. 2019a,b).

\footnotetext{
Antioxidant enzyme activities and roles in leaf senescence: Leaf senescence results in the accumulation of high amounts of reactive oxygen species (ROS) and the activities of antioxidant enzymes can reduce the ROS contents in plants (El Hadrami et al. 2005, Li et al. 2007). Our previous study of wheat showed that UCZ increased the antioxidant enzyme activities and the higher activity levels decreased the accumulation of ROS (Ahmad et al. $2019 b$ ). Triazoles may increase the antioxidant enzyme
}

activities and reduce the ROS contents in wheat, rice, and winter rape (Zhang et al. 2010, Pan et al. 2013). Our results showed that the activity of SOD increased from V3 to R1-15 and then decreased. The activity of POD increased from V3 to R1-30 and then decreased. The activities of CAT and APX were higher at V3, decreased at V8, and then they tended to increase until maturity. The application of UCZ together with $\mathrm{N}$ significantly increased the antioxidant enzyme activities, where the maximum values were obtained under $\mathrm{U}_{25} \mathrm{~N}_{150}$ and the minimum values under $\mathrm{U}_{0} \mathrm{~N}_{0}$. We also found that the application of $\mathrm{N}$ alone increased the antioxidant enzyme activities and reduced leaf senescence. The higher antioxidant enzyme activities protected the $\mathrm{Chl}$ pigments to enhance the photosynthetic efficiency and reduce the leaf senescence rate in the high density plant population. The application of $\mathrm{N}$ at a rate of $225 \mathrm{~kg} \mathrm{ha}^{-1}$ decreased the leaf area per plant, Chl contents, SP contents, $P_{\mathrm{N}}, g_{\mathrm{s}}, E, \mathrm{ETR}, \mathrm{q}_{\mathrm{p}}, \mathrm{F}_{\mathrm{v}} / \mathrm{F}_{\mathrm{m}}, \Phi_{\mathrm{PSII}}, \mathrm{F}_{\mathrm{v}}$, antioxidant enzyme activities, yield, and yield components compared to those when $\mathrm{N}$ was applied at $150 \mathrm{~kg} \mathrm{ha}^{-1}$, probably due to the inhibitory effect of the higher $\mathrm{N}$ rate. In addition, the higher $\mathrm{N}$ rate may have resulted in taller plants with a higher lodging rate. However, this difference requires further investigation.

Effects of UCZ and $N$ fertilization on grain yield of maize crop: The application of UCZ together with $\mathrm{N}$ significantly increased the maize grain yield under the high density plant population. The higher grain yield under UCZ together with $\mathrm{N}$ in the high density plant population was attributable to the higher Chl contents, photosynthetic efficiency, and Chl fluorescence, and the enhanced antioxidant defense system. The application of UCZ together with $\mathrm{N}$ protected the photosynthetic system and obtained higher grain yields. Previous studies also showed that the application of UCZ together with $\mathrm{N}$ increased the grain yield in various crops by reducing the lodging rate and enhancing the photosynthetic performance (Wang et al. 2009, 2015a; Su et al. 2019). In addition, our previous studies demonstrated that UCZ increased the grain yield in maize with a medium plant population density by reducing leaf senescence in the bottom layer. In the present study, the application of UCZ at a concentration of $25 \mathrm{mg} \mathrm{kg}^{-1}$ and nitrogen at a rate of $150 \mathrm{~kg} \mathrm{ha}^{-1}$ resulted in the maximum grain yield in a high density plant population.

Conclusion: In the present study, we found that the application of UCZ and $\mathrm{N}$ fertilizer significantly increased the maize grain yield under high density plant population. The application of UCZ and N increased the leaf area per plant, chlorophyll $a$ and $b$ contents, $P_{\mathrm{N}}, g_{\mathrm{s}}$, and $E$ but reduced $C_{\mathrm{i}}$. Applying UCZ together with $\mathrm{N}$ also increased ETR, $\mathrm{q}_{\mathrm{p}}, \mathrm{F}_{\mathrm{v}} / \mathrm{F}_{\mathrm{m}}, \Phi_{\mathrm{PSI}}$, and $\mathrm{F}_{\mathrm{v}}$ but reduced $\mathrm{q}_{\mathrm{N}}$. Furthermore, UCZ together with $\mathrm{N}$ enhanced the SP content and antioxidant enzyme activities but reduced the MDA content. The lower MDA content and higher antioxidant enzyme activities protected the photosynthetic pigments and increased the leaf area per plant. Compared to the application of $\mathrm{N}$ alone, applying UCZ together with $\mathrm{N}$ increased the leaf area per plant, chlorophyll $a$ and $b$ contents, $P_{\mathrm{N}}, g_{\mathrm{s}}, E$, 
ETR, $\mathrm{q}_{\mathrm{p}}, \mathrm{F}_{\mathrm{v}} / \mathrm{F}_{\mathrm{m}}, \Phi_{\mathrm{PSII}}$, and $\mathrm{F}_{\mathrm{v}}$ but decreased $C_{\mathrm{i}}, \mathrm{q}_{\mathrm{N}}$, and the MDA content. The application of UCZ together with $\mathrm{N}$ decreased the degradation of chlorophyll and reduced the leaf senescence rate under high density plant population, and $\mathrm{U}_{25} \mathrm{~N}_{150}$ obtained the maximum grain yield of 13,728 $\mathrm{kg} \mathrm{ha}^{-1}$.

\section{References}

Aebi H.: Catalase in vitro. - Method. Enzymol. 105: 121-126, 1984.

Ahmad I., Kamran M., Ali S. et al.: Uniconazole application strategies to improve lignin biosynthesis, lodging resistance and production of maize in semiarid regions. - Field Crop. Res. 222: 66-77, 2018a.

Ahmad I., Kamran M., Ali S. et al.: Seed filling in maize and hormones crosstalk regulated by exogenous application of uniconazole in semiarid regions. - Environ. Sci. Pollut. R. 25: 33225-33239, 2018b.

Ahmad I., Kamran M., Guo Z.Y. et al.: Effects of uniconazole or ethephon foliar application on culm mechanical strength and lignin metabolism, and their relationship with lodging resistance in winter wheat. - Crop Pasture Sci. 71: 12-22, 2020.

Ahmad I., Kamran M., Meng X.P. et al.: Hormonal changes with uniconazole triggers canopy apparent photosynthesis and grain filling in wheat crop in a semi-arid climate. Protoplasma 258: 139-150, 2021.

Ahmad I., Kamran M., Su W. et al.: Application of uniconazole improves photosynthetic efficiency of maize by enhancing the antioxidant defense mechanism and delaying leaf senescence in semiarid regions. - J. Plant Growth Regul. 38: 855-869, 2019a.

Ahmad I., Kamran M., Yang X.N. et al.: Effects of applying uniconazole alone or combined with manganese on the photosynthetic efficiency, antioxidant defense system, and yield in wheat in semiarid regions. - Agr. Water Manage. 216: 400-414, 2019b.

Ajigboye O.O., Murchie E., Ray R.V.: Foliar application of isopyrazam and epoxiconazole improves photosystem II efficiency, biomass and yield in winter wheat. - Pestic. Biochem. Phys. 114: 52-60, 2014.

Ali S., Xu Y.Y., Jia Q.M. et al.: Ridge-furrow mulched with plastic film improves the anti-oxidative defence system and photosynthesis in leaves of winter wheat under deficit irrigation. - PLoS ONE 13: e0200277, 2018.

Bradford M.M.: A rapid and sensitive method for the quantization of microgram quantities of protein utilizing the principle of protein dye binding. - Anal. Biochem. 72: 248-254, 1976.

Davis T.D., Steffens G.L., Sankhla N.: Triazole plant growth regulators. - In: Janick J. (ed.): Horticultural Reviews. Vol. 10. Pp. 63-105. Timber Press, Portland 1988.

Dong H.Z., Li W.J., Eneji A.E., Zhang D.M.: Nitrogen rate and plant density effects on yield and late-season leaf senescence of cotton raised on a saline field. - Field Crop. Res. 126: 137144, 2012.

Dordas C.A., Sioulas C.: Safflower yield, chlorophyll content, photosynthesis, and water use efficiency response to nitrogen fertilization under rainfed conditions. - Ind. Crop. Prod. 27: $75-85,2008$

Duan L., Guan C., Li J. et al.: Compensative effects of chemical regulation with uniconazole on physiological damages caused by water deficiency during the grain filling stage of wheat. J. Agron. Crop Sci. 194: 9-14, 2008.

El Hadrami A., Kone D., Lepoivre P.: Effect of juglone on active oxygen species and antioxidant enzymes in susceptible and partially resistant banana cultivars to black leaf streak disease. - Eur. J. Plant Pathol. 113: 241-254, 2005.

Fang X., Li Y., Nie J. et al.: Effects of nitrogen fertilizer and planting density on the leaf photosynthetic characteristics, agronomic traits and grain yield in common buckwheat (Fagopyrum esculentum M.). - Field Crop. Res. 219: 160$168,2018 \mathrm{a}$.

Fang X., Liu X., Zhang Y. et al.: Effects of uniconazole or gibberellic acid application on the lignin metabolism in relation to lodging resistance of culm in common buckwheat (Fagopyrum esculentum M.). - J. Agron. Crop Sci. 204: 414423, 2018b.

Frymire R.M., Cole J.C.: Uniconazole effect on growth and chlorophyll content of pyracantha, photinia, and dwarf Burford holly. - J. Plant Growth Regul. 11: 143-148, 1992.

Giannopolitis C.N., Ries S.K.: Superoxide dismutases I. Occurrence in higher plants. - Plant Physiol. 59: 309-314, 1977.

Gregersen P.L.: Senescence and nutrient remobilization in crop plants. - In: Hawkesford M.J., Barraclough P. (ed.): The Molecular and Physiological Basis of Nutrient Use Efficiency in Crops. ${ }^{\text {st }}$ Edition. Pp. 83-102. Wiley Blackwell, Chichester 2011.

Gregersen P.L., Culetic A., Boschian L., Krupinska K.: Plant senescence and crop productivity. - Plant Mol. Biol. 82: 603622, 2013.

Hernández J.A., Jiménez A., Mullineaux P., Sevilia F.: Tolerance of pea (Pisum sativum L.) to long-term salt stress is associated with induction of antioxidant defences. - Plant Cell Environ. 23: 853-862, 2000.

Hubert B., Rosengrant M., van Boekel M.A.J.S., Ortiz R.: The future of food: Scenarios for 2050. - Crop Sci. 50: S-33-S-50, 2010.

Jaleel C.A., Gopi R., Azooz M.M., Panneerselvam R.: Leaf anatomical modification in Cataranthus roseus as affected by plant growth promotors and retardants. - Glob. J. Mol. Sci. 4: $1-5,2009$.

Kamran M., Ahmad I., Wang H.Q. et al.: Mepiquat chloride application increases lodging resistance of maize by enhancing stem physical strength and lignin biosynthesis. - Field Crop. Res. 224: 148-159, 2018.

Kitonyo O.M., Sadras V.O., Zhou Y., Denton M.D.: Nitrogen supply and sink demand modulate the patterns of leaf senescence in maize. - Field Crop. Res. 225: 92-103, 2018.

Li G., Zhang Z.S., Gao H.Y. et al.: Effects of nitrogen on photosynthetic characteristics of leaves from two different stay-green corn (Zea mays L.) varieties at the grain-filling stage. - Can. J. Plant Sci. 92: 671-680, 2012.

Li T., Zhang Y.J., Dai J.L. et al.: High plant density inhibits vegetative branching in cotton by altering hormone contents and photosynthetic production. - Field Crop. Res. 230: 121131, 2019.

Li Z.Z., Niu W., Qiao X.W., Ping M.L.: Anti-oxidant response of Cucumis sativus L. to fungicide carbendazim. - Pestic. Biochem. Phys. 89: 54-59, 2007.

Lin Y.C., Hu Y.G., Ren C.Z. et al.: Effects of nitrogen application on chlorophyll fluorescence parameters and leaf gas exchange in naked oat. - J. Integr. Agr. 12: 2164-2171, 2013.

Liu F., Jensen C.R., Andersen M.N.: A review of drought adaptation in crop plants: changes in vegetative and reproductive physiology induced by ABA-based chemical signals. - Aust. J. Agr. Res. 56: 1245-1252, 2005.

Luo Z., Liu H., Li W.P. et al.: Effects of reduced nitrogen rate on cotton yield and nitrogen use efficiency as mediated by application mode or plant density. - Field Crop. Res. 218: 
$150-157,2018$

Maddonni G., Otegui M.E., Cirilo A.G.: Plant population density, row spacing and hybrid effects on maize canopy architecture and light attenuation. - Field Crop. Res. 71: 183-193, 2001.

Mu H., Jiang D., Wollenweber B. et al.: Long-term low radiation decreases leaf photosynthesis, photochemical efficiency and grain yield in winter wheat. - J. Agron. Crop Sci. 196: 38-47, 2010.

Nakano Y., Asada K.: Hydrogen peroxide is scavenged by ascorbate-specific peroxidase in spinach chloroplasts. - Plant Cell Physiol. 22: 867-880, 1981.

Pan S., Rasul F., Li W. et al.: Roles of plant growth regulation on yield, grain qualities and antioxidant enzyme activities in super hybrid rice (Oryza sativa L.). - Rice 6: 9, 2013.

Sadras V.O., Echarte L., Andrade F.A.: Profiles of leaf senescence during reproductive growth of sunflower and maize. - Ann. Bot.-London 85: 187-195, 2000.

Sage R.F., Pearcy R.W.: The nitrogen use efficiency of $\mathrm{C}_{3}$ and $\mathrm{C}_{4}$ plants II: leaf nitrogen effects on the gas exchange characteristics of Chenopodium album (L.) and Amaranthus retroflexus (L.). - Plant Physiol. 84: 959-963, 1987.

Shangguan Z., Shao M., Dyckmans J.: Effects of nitrogen nutrition and water deficit on net photosynthetic rate and chlorophyll fluorescence in winter wheat. - J. Plant Physiol. 156: 46-51, 2000.

Shrestha S., Brueck H.,Asch F.: Chlorophyll index: photochemical reflectance index and chlorophyll fluorescence measurements of rice leaves supplied with different $\mathrm{N}$ levels. - J. Photoch. Photobio. B 113: 7-13, 2012.

Soumya P.R., Pramod K., Madan P.: Paclobutrazol: A novel plant growth regulator and multi-stress ameliorant. - Ind. J. Plant Physiol. 22: 267-278, 2017.

Su W.N., Kamran M., Xie J. et al.: Shoot and root traits of summer maize hybrid varieties with higher grain yields and higher nitrogen use efficiency at low nitrogen application rates. - PeerJ 7: e7294, 2019.

Thomas H., Ougham H.: Senescence and crop performance. In: Sadras V.O., Calderini D.F. (ed.), Crop Physiology: Applications for Genetic Improvement and Agronomy. Pp. 223-249. Elsevier, London 2015.

van Doorn W.G., Woltering E.J.: Senescence and programmed cell death: substance or semantics? - J. Exp. Bot. 55: 21472153, 2004.

Wang C., Hu D., Liu X.B. et al.: Effects of uniconazole on the lignin metabolism and lodging resistance of culm in common buckwheat (Fagopyrum esculentum M.). - Field Crop. Res. 180: 46-53, 2015a.

Wang P., Sun X., Li C. et al.: Long-term exogenous application of melatonin delays drought-induced leaf senescence in apple. - J. Pineal Res. 54: 292-302, 2013.

Wang X.C., Yang W.Y., Chen G. et al.: Effects of uniconazole on leaf senescence and yield of maize sprayed at late growth stage. - J. Maize Sci. 17: 86-88, 2009. [In Chinese]

Wang Y.C., Gu W.R., Ye L.F. et al.: Physiological mechanism of delaying leaf senescence in maize treated with compound mixtures of DCPTA and CCC. - J. Northeast Agr. Univ. 22: $1-15,2015 b$.

Wei S.S., Wang X.Y., Shi D.Y. et al.: The mechanisms of low nitrogen induced weakened photosynthesis in summer maize (Zea mays L.) under field conditions. - Plant Physiol. Bioch. 105: 118-128, 2016.

Wu X.Y., Kuai B.K., Jia J.Z., Jing H.C.: Regulation of leaf senescence and crop genetic improvement. - J. Integr. Plant Biol. 54: 936-952, 2012.

Wu Y.W., Li Q., Jin R. et al.: Effect of low-nitrogen stress on photosynthesis and chlorophyll fluorescence characteristics of maize cultivars with different low nitrogen tolerances. J. Integr. Agr. 18: 1246-1256, 2019.

Xia X., Tang J.H., Wei M.R., Zhao D.Q.: Effect of paclobutrazol application on plant photosynthetic performance and leaf greenness of herbaceous peony. - Horticulturae 4: 5, 2018.

Xu C., Gao Y., Tian B. et al.: Effects of EDAH, a novel plant growth regulator, on mechanical strength, stalk vascular bundles and grain yield of summer maize at high densities. Field Crop. Res. 200: 71-79, 2017.

Xue J., Xie R.Z., Zhang W.F. et al.: Research progress on reduced lodging of high-yield and -density maize. - J. Integr. Agr. 16: 2717-2725, 2017.

Yu J.Q., Huang L.F., Hu W.H. et al.: A role for brassinosteroids in the regulation of photosynthesis in Cucumis sativus. - J. Exp. Bot. 55: 1135-1143, 2004.

Zhang J., Cao X.L., Yong T.W. et al.: Seed treatment with uniconazole powder induced drought tolerance of soybean in relation to changes in photosynthesis and chlorophyll fluorescence. - Res. Crop. 13: 147-154, 2012.

Zhang M., Duan L., Tian X. et al.: Uniconazole-induced tolerance of soybean to water deficit stress in relation to changes in photosynthesis, hormones and antioxidant system. - J. Plant Physiol. 164: 709-717, 2007.

Zhang Y.J., Zhang X., Chen C.J. et al.: Effects of fungicides JS399-19, azoxystrobin, tebuconazole, and carbendazim on the physiological and biochemical indices and grain yield of winter wheat. - Pestic. Biochem. Phys. 98: 151-157, 2010.

Zhao H., Dai T., Jing Q. et al.: Leaf senescence and grain filling affected by post-anthesis high temperatures in two different wheat cultivars. - Plant Growth Regul. 51: 149-158, 2007.

Zhong X., Che X., Zhang Z. et al.: Slower development of PSI activity limits photosynthesis during Euonymus japonicus leaf development. - Plant Physiol. Bioch. 136: 13-21, 2019.

Zivcak M., Brestic M., Balatova Z. et al.: Photosynthetic electron transport and specific photo protective responses in wheat leaves under drought stress. - Photosynth. Res. 117: 529-546, 2013.

(C) The authors. This is an open access article distributed under the terms of the Creative Commons BY-NC-ND Licence. 